\title{
Contingency-Constrained Unit Commitment with Post-Contingency Corrective Recourse
}

\author{
Richard Li-Yang Chen · Neng Fan · Ali Pinar · \\ Jean-Paul Watson
}

Received: date / Accepted: date

\begin{abstract}
We consider the problem of minimizing costs in the generation unit commitment problem, a cornerstone in electric power system operations, while enforcing an $N-k-\varepsilon$ reliability criterion. This reliability criterion is a generalization of the wellknown $N-k$ criterion and dictates that at least $(1-\varepsilon)$ fraction of the total system demand must be met following the failure of $k$ or fewer system components. We refer to this problem as the Contingency-Constrained Unit Commitment problem, or CCUC. We present a mixed-integer programming formulation of the CCUC that accounts for both transmission and generation element failures. We propose novel cutting plane algorithms that avoid the need to explicitly consider an exponential number of contingencies. Computational studies are performed on several IEEE test systems and a simplified model of the Western US interconnection network. These studies demonstrate the effectiveness of our proposed methods relative to current state-of-the-art.
\end{abstract}

Keywords Integer programming · Bi-level programming · Benders decomposition · Unit commitment $\cdot$ Contingency constraints

\section{Introduction}

Power system operations aim to optimally utilize available electricity generation resources to satisfy projected demand, at minimal cost, subject to various physical generation and transmission constraints, and operational security constraints. Tradition-

R.L.-Y. Chen · A. Pinar

Quantitative Modeling and Analysis, Sandia National Laboratories, Livermore, CA 94551

E-mail: \{rlchen,apinar\}@sandia.gov

N. Fan

Department of Systems and Industrial Engineering, University of Arizona, Tucson, AZ 85721

E-mail: nfan@email.arizona.edu

J.-P. Watson

Analytics Department, Sandia National Laboratories, Albuquerque, NM 87185

E-mail: jwatson@sandia.gov 
ally, such operations involve numerous sub-tasks, including short-term load forecasting, unit commitment, economic dispatch, voltage and frequency control, and interchange scheduling between distinct electricity grid operators. Most recently, renewable generation units in the form of geographically distributed wind and solar farms have imposed the additional requirement to consider uncertain generation output, increasingly in conjunction with the deployment of advanced storage technologies such as pumped hydro. Growth in system size and the introduction of significant generation output uncertainty contribute to increased concerns regarding system vulnerability. Large-scale blackouts, such as the Northeast blackout of 2003 in North America and, more recently, the blackout of July 2012 in India, impact millions of people and result in significant economic costs. Similarly, failure to accurately account for renewables output uncertainty can lead to large-scale forced outages, as in the case of ERCOT (a major electricity grid operator in Texas) on February 26, 2008. Such events have led to an increased focus on power systems reliability, with the goal of mitigating against failures due to both natural causes and intelligent adversaries.

Optimization methods have been applied to power system operations problems for several decades; Wood and Wollenberg [30] provide a brief overview. The coupling of state-of-the-art implementations of core optimization algorithms (including simplex, barrier, and mixed-integer branch-and-cut algorithms) and current computing capabilities (e.g., inexpensive multi-core processors) enable optimal decisionmaking in real power systems. One notable example involves the unit commitment problem, which is used to determine the day-ahead schedule for all generators in a given operating region of an electricity grid. A solution to the unit commitment problem specifies, for each hour in the scheduling horizon (typically 24 or 48 hours), both the set of generators that are to be operating and their corresponding projected power output levels. The solution must satisfy a large number of generator (e.g., ramp rates, minimum up and down times, and capacity limits) and transmission (e.g., power flow and thermal limit) constraints, achieving a minimal total production cost while satisfying forecasted demand. The unit commitment problem has been widely studied, for over three decades. For a review of the relevant literature, we refer to [13] and the more recent [21]. Many heuristic (e.g., genetic algorithms, tabu search, and simulated annealing) and mathematical (e.g., integer programming, dynamic programming, and Lagrangian relaxation) optimization methods have been introduced to solve the unit commitment problem. Until the early 2000s, Lagrangian relaxation methods were the dominant approach used in practice. However, mixed-integer programming implementations are either currently in use or are scheduled to be adopted by all Independent System Operators (ISOs) in the United States to solve their unit commitment problems [9].

Security constraints - which ensure that system performance is sustained when certain components fail - in the context of unit commitment are now a required regulatory element of power systems operations. The North American Electric Reliability Corporation (NERC) develops and enforces standards to ensure power systems reliability in North America. Of strongest relevance to security constraints for unit commitment is the NERC Transmission Planning Standard (TPL-001-0.1, TPL-0020b, TPL-003-0b, TPL-004-0a, [18]). The TPL specifies four categories of operating states, labeled A through D. Category A represents the baseline "normal" state, during 
which there are no system component failures. Category B represents so called $N-1$ contingency states, in which a single system component has failed (out of a total of $N$ components, including generators and transmission lines). NERC requires no lossof-load in both categories $\mathrm{A}$ and $\mathrm{B}$, which collectively represent the vast majority of observed operational states. Categories $\mathrm{C}$ and D of the TPL represent more extreme states, in which multiple system components fail (near) simultaneously. Large-scale blackouts, typically caused by cascading failures, are Category D events. Such failure states are known as $N-k$ contingencies in the power system literature, where $k(k \geq 2)$ denotes the number of component failures. In contrast to categories $\mathrm{A}$ and $\mathrm{B}$, the regulatory requirements for categories C and D are vaguely specified, e.g., "some" loss of load is allowable, and it is permissible to exceed normal transmission line capacities by unspecified amounts for brief time periods.

The computational difficulty of security-constrained unit commitment is wellknown, and is further a function of the specific TPL category that is being considered. The unit commitment problem subject to $N-1$ reliability constraints is, given the specific regulatory requirements imposed for category B events of the TPL, addressed by system operators worldwide. However, we observe that this problem is often solved approximately in practice, specifically in the context of large-scale (ISO-scale) systems [20]. For example, a subset of contingencies based on a careful engineering analysis is often used to obtain a computationally tractable unit commitment problem. Alternatively, the unit commitment problem can be solved without considering contingencies, and the solution can be subsequently "screened" for validity under a subset of contingencies (again identified by engineering analysis). Additional constraints can then be added to the master unit commitment problem, which is then re-solved; the process repeats until there is no loss-of-load in the contingency states. We raise this issue primarily to point out that even the full $N-1$ problem is not considered a "solved" problem in practice, such that advances (including those introduced in this paper) in the solution of unit commitment problems subject to general $\mathrm{N}-k$ reliability constraints can potentially impact the practical solution of the simpler $N-1$ problem variant.

Numerous researchers have introduced algorithms for solving both the securityconstrained unit commitment problem and the simpler, related problem of securityconstrained optimal power flow. In the latter, the analysis is restricted to a single time period, and binary variables relating to generation unit status are fixed based on a pre-computed unit commitment schedule. Reference [4] provides a recent review of the literature on security-constrained optimal power flow. Of specific relevance to our work is the literature on security-constrained optimal power flow in situations where large numbers of system components fail. This literature is mostly based on worstcase network interdiction analysis and includes solution methods based on bi-level and mixed-integer programming (see $[24,25,1,8,33,32])$ and graph algorithms (see $[22,3,8,15,16])$.

Following the Northeast US blackout of 2003, significant attention was focused on developing improved solution methods for the security-constrained unit commitment problem. In particular, various researchers introduced mixed-integer programming and decomposition-based methods for more efficiently enforcing $N$-1 reliability, e.g., see $[10,11,28,17,12,19]$. However, due to its computational complexity, 
security-constrained unit commitment considering the full spectrum of NERC reliability standards has not attracted a comparable level of attention until very recently. Specifically, [26] and [29] consider the case of security-constrained unit commitment under the more general $N-k$ reliability criterion. Similarly, [26,27] and [29] use robust optimization methods for identifying worst-case $N-k$ contingencies.

In this paper, we extend the $N-k$ reliability criterion to yield the more general $N-k$ $\varepsilon$ criterion. This new criterion dictates that for all contingencies of size $j \in\{1, \cdots, k\}$, at least $\left(1-\varepsilon_{j}\right)$ fraction of the total demand must be met, with $0 \leq \varepsilon_{1} \leq \varepsilon_{2} \leq \cdots \leq$ $\varepsilon_{k} \leq 1$. The primary motivation for introducing this variant of the $N-k$ metric is that it provides a practical and quantifiable bound on system performance under Category $\mathrm{C}$ and D TPL contingencies, and can easily be expressed in mathematical optimization models. We refer to the security-constrained unit commitment problem subject to $N-k-\varepsilon$ reliability as the contingency-constrained unit commitment (CCUC) problem. In the CCUC, all contingencies with $k$ or fewer system element failures (generation units or transmission lines) are implicitly considered when checking for the feasibility of post-contingency corrective recourse. The CCUC is formulated as a large-scale mixed-integer linear program (MILP). To solve the CCUC, we develop two decomposition strategies: one based on a Benders decomposition [2], and another based on cutting planes derived from the solution of power system inhibition problems [6, 7]. We then show the computational effectiveness of our algorithms on a range of benchmark instances.

Our specific contributions, as detailed in this paper, include:

- We ensure the existence of a feasible post-contingency corrective recourse, taking into consideration generator ramping constraints and the no-contingency (nominal) state economic dispatch;

- We consider the losses of both generation units and transmission lines;

- We propose novel decomposition methods to solve the contingency-constrained CCUC efficiently, and show that models and methods proposed by [12], [19], [26], [27], and [29] are all special cases of our general approach.

The remainder of this paper is organized as follows. In Section 2, we formulate the MILP model for the contingency-constrained unit commitment problem under the $N-k-\varepsilon$ reliability criterion. In Section 3, two approaches based on decomposition methods are presented for solving this large-scale MILP. In Section 4, we test our algorithms on several IEEE test systems and a simplified model of the Western interconnection. Finally, we conclude in Section 5 with a summary of our results and directions for future research.

\section{Problem Formulation}

In this section, we present our mixed-integer linear programming model for the contingencyconstrained unit commitment (CCUC) problem. In Table 1, we introduce the core sets, parameters, and decision variables of the model. The baseline unit commitment formulation, without contingency constraints, is described in Section 2.1. We discuss key concepts involving $N-k-\varepsilon$ contingency analysis in Section 2.2, which are subsequently illustrated using an example in Section 2.3. Finally, we combine the baseline 
Table 1 Nomenclature

\begin{tabular}{|c|c|}
\hline \multicolumn{2}{|r|}{ Sets and Indices } \\
\hline $\mathscr{I}$ & Set of buses. Indexed by $i$ for individual buses, $i$ and $j$ for pairs of buses. \\
\hline$I$ & Number of buses. $I=|\mathscr{I}|$ \\
\hline $\mathscr{G}$ & Set of generation units. Indexed by $g$. \\
\hline$G$ & Number of generation units. $G=|\mathscr{G}|$. \\
\hline $\mathscr{G}_{i}$ & Set of generation units located at bus $i \in \mathscr{I}$ \\
\hline $\mathscr{E}$ & Set of directed transmission lines connecting pairs of buses. Indexed by $e$. \\
\hline$E$ & Number of directed transmission lines. $E=|\mathscr{E}|$ \\
\hline $\mathscr{E}_{. i}$ & Set of transmission lines oriented into bus $i \in \mathscr{I}$. \\
\hline $\mathscr{E}_{i}$ & Set of transmission lines oriented out of bus $i \in \mathscr{I}$. \\
\hline$i_{e}, j_{e}$ & Tail bus $i_{e}$ and head bus $j_{e}$ of transmission line $e \in \mathscr{E}$. \\
\hline $\begin{array}{c}\mathscr{T} \\
(\mathscr{I}, \mathscr{G}, \mathscr{E})\end{array}$ & $\begin{array}{l}\text { Set of time periods in the planning horizon. Indexed by } t \in\{1,2, \cdots, T\} \text {. } \\
\text { triple that defines a power system. }\end{array}$ \\
\hline \multicolumn{2}{|r|}{ Parameters } \\
\hline$B_{e}, F_{e}$ & Susceptance and power flow (i.e., thermal) limit of transmission line $e$. \\
\hline$D_{i}^{l}$ & Demand (load) at bus $i \in \mathscr{I}$ at time period $t$. \\
\hline$D^{t}=\sum_{i \in \mathscr{I}} D_{i}^{t}$ & Total demand, across all buses, in time period $t$. \\
\hline$P_{g}^{\min }, P_{g}^{\max }$ & Lower/upper limits on power output for generation unit $g$. \\
\hline$T_{g}^{d 0}, T_{g}^{u 0}$ & Minimum time periods generation unit $g \in \mathscr{G}$ must be initially offline/online. \\
\hline$T_{g}^{d}, T_{g}^{u}$ & $\begin{array}{l}\text { Minimum time periods generation unit } g \in \mathscr{G} \text { must remain offline/online once the unit } \\
\text { is shut down/started up. }\end{array}$ \\
\hline$R_{g}^{d}, R_{g}^{u}$ & $\begin{array}{l}\text { Maximum ramp-down and ramp-up rate for generation unit } g \in \mathscr{G} \text { between adjacent } \\
\text { time periods. }\end{array}$ \\
\hline$\tilde{R}_{g}^{d}, \tilde{R}_{g}^{u}$ & $\begin{array}{l}\text { Maximum shutdown/startup ramp rates for generation unit } g \in \mathscr{G} \text { for a time period } \\
\text { in which } g \text { is turned off/on. }\end{array}$ \\
\hline$C_{g}^{u}, C_{g}^{d}$ & Fixed startup/shutdown cost for generation unit $g$. \\
\hline$C_{g}^{p}(\cdot)$ & Production cost function for generation unit $g$. \\
\hline \multicolumn{2}{|r|}{ Variables } \\
\hline$x_{g}^{t}$ & $\begin{array}{l}\text { Binary variable indicating if a generation unit } g \in \mathscr{G} \text { is committed }\left(x_{g}^{t}=1\right) \text { or not } \\
\left(x_{g}^{t}=0\right) \text { at time } t \text {. }\end{array}$ \\
\hline $\mathbf{x}^{t}$ & Unit commitment decision vector for all generation units at time $t$. \\
\hline $\mathbf{x}$ & Length $G \times T$ unit commitment decision vector. \\
\hline$c_{g}^{u t}, c_{g}^{d t}$ & $\begin{array}{l}\text { Incurred startup/shutdown cost for a generation unit } g \in \mathscr{G} \text { at time } t \text { (if unit } g \text { is started } \\
\text { up or shut down at time } t \text {, the respective costs are } C_{g}^{u} \text { and } C_{g}^{d} \text {. Otherwise, } C_{g}^{u}=C_{g}^{d}=0 \text {.) }\end{array}$ \\
\hline $\mathbf{c}^{u}, \mathbf{c}^{d}$ & Length $G \times T$ startup/shutdown cost decision vectors. \\
\hline$\tilde{p}_{g}^{t}$ & No-contingency state power output by generation unit $g$ at time $t$. \\
\hline$\tilde{f}_{e}^{t}$ & No-contingency state power flow on transmission line $e$ at time $t$. \\
\hline$\tilde{\theta}_{i}^{t}$ & No-contingency state phase angle at bus $i$ at time $t$. \\
\hline$\tilde{p}$ & Length $G \times T$ power output vector. \\
\hline$\tilde{f}$ & Length $E \times T$ power flow vector. \\
\hline$\tilde{\theta}$ & Length $E \times T$ phase angle vector. \\
\hline
\end{tabular}

unit commitment model with $N-k-\varepsilon$ contingency analysis in Section 2.4, to form our contingency-constrained unit commitment model.

\subsection{The Baseline Unit Commitment Model}

We now present our baseline unit commitment (BUC) formulation, without contingency constraints. Our formulation is based on the mixed-integer linear programming 
UC formulations introduced by $[5,31,34]$. We extend these formulations to capture network transmission constraints, in the form of a DC power flow model. Our BUC model is intended to reflect steady-state operational conditions, such that the system is in a no-contingency state. Consequently, we require that the demand at each bus $i \in \mathscr{I}$ must be fully satisfied, i.e., no loss-of-load is allowed.

Our BUC formulation for a power system $(\mathscr{I}, \mathscr{G}, \mathscr{E})$ is given as follows:

$$
\begin{aligned}
& \min _{\mathbf{x}, \mathbf{c}^{u}, \mathbf{c}^{d}} \sum_{t \in \mathscr{T}} \sum_{g \in \mathscr{G}}\left(c_{g}^{u t}+c_{g}^{d t}\right)+\mathscr{Q}(\mathbf{x}) \\
& \text { s.t. } \sum_{t=1}^{T_{g}^{u 0}}\left(1-x_{g}^{t}\right)=0 \\
& \sum_{t=1}^{T_{g}^{d 0}} x_{g}^{t}=0 \\
& \sum_{t^{\prime}=t}^{t+T_{g}^{u}-1} x_{g}^{t^{\prime}} \geq T_{g}^{u}\left(x_{g}^{t}-x_{g}^{t-1}\right) \quad \forall g \in \mathscr{G}, t \in\left\{T_{g}^{u 0}+1, \cdots, T-T_{g}^{u}+1\right\} \\
& \sum_{t^{\prime}=t}^{T}\left(x_{g}^{t^{\prime}}-\left(x_{g}^{t}-x_{g}^{t-1}\right)\right) \geq 0 \\
& \forall g \in \mathscr{G}, t \in\left\{T-T_{g}^{u}+2, \cdots, T\right\}
\end{aligned}
$$

$$
\begin{array}{ll}
\sum_{t^{\prime}=t}^{t+T_{g}^{d}-1}\left(1-x_{g}^{t^{\prime}}\right) \geq T_{g}^{d}\left(x_{g}^{t-1}-x_{g}^{t}\right) & \forall g \in \mathscr{G}, t \in\left\{T_{g}^{d 0}+1, \cdots, T-T_{g}^{d}+1\right\} \\
\sum_{t^{\prime}=t}^{T}\left(\left(1-x_{g}^{t^{\prime}}\right)-\left(x_{g}^{t-1}-x_{g}^{t}\right)\right) \geq 0 & \forall g \in \mathscr{G}, t \in\left\{T-T_{g}^{d}+2, \cdots, T\right\} \\
c_{g}^{u t} \geq C_{g}^{u}\left(x_{g}^{t}-x_{g}^{t-1}\right) & \forall g \in \mathscr{G}, t \in \mathscr{T} \\
c_{g}^{d t} \geq C_{g}^{d}\left(x_{g}^{t-1}-x_{g}^{t}\right) & \forall g \in \mathscr{G}, t \in \mathscr{T} \\
c_{g}^{u t}, c_{g}^{d t} \geq 0 & \forall g \in \mathscr{G}, t \in \mathscr{T} \\
x_{g}^{t} \in\{0,1\} & \forall g \in \mathscr{G}, t \in \mathscr{T}
\end{array}
$$

The optimization objective (1a) is to minimize the sum of the startup costs $c_{g}^{u t}$, shutdown costs $c_{g}^{d t}$, and generation cost $\mathscr{Q}(\mathbf{x})$. Constraints (1b) - (1k) include (in order): initial online requirements for generation units (1b); initial offline requirements 
for generation units (1c); minimum online constraints in nominal time periods for generation units (1d); minimum online constraints for the last $T_{g}^{u}$ time periods (1e); minimum offline constraints in nominal time periods for generation units (1f); minimum offline constraints for the last $T_{g}^{d}$ time periods (1g); startup cost computation (1h); shutdown cost computation (1i); non-negativity constraints for startup and shutdown costs (1j); and binary constraints for the on/off status of generation units (1k). For clarity of exposition and conciseness, we denote the set of feasible commitments by $\mathscr{X}=\left\{\left(\mathbf{x}, \mathbf{c}^{d}, \mathbf{c}^{u}:\right.\right.$ Constraints $\left.(1 \mathrm{~b})-(1 \mathrm{k})\right\}$.

The minimum generation cost $\mathscr{Q}(\mathbf{x})$, given a fixed unit commitment $\mathbf{x}$, is constrained by a combination of DC power flow constraints and unit ramping constraints, given as follows:

$$
\begin{aligned}
& \mathscr{Q}(\mathbf{x})=\min _{\tilde{\mathbf{f}}, \tilde{\mathbf{p}}, \tilde{\theta}} \sum_{g \in \mathscr{G}} \sum_{t \in \mathscr{T}} C_{g}^{p}\left(\tilde{p}_{g}^{t}\right) \\
& \text { s.t. } \quad \sum_{g \in \mathscr{G}_{i}} \tilde{p}_{g}^{t}+\sum_{e \in \mathscr{E}_{. i}} \tilde{f}_{e}^{t}-\sum_{e \in \mathscr{E}_{i .}} \tilde{f}_{e}^{t}=D_{i}^{t} \\
& B_{e}\left(\tilde{\theta}_{i_{e}}^{t}-\tilde{\theta}_{j_{e}}^{t}\right)-\tilde{f}_{e}^{t}=0 \quad \forall e \in \mathscr{E} \\
& -F_{e} \leq \tilde{f}_{e}^{t} \leq F_{e} \\
& \forall e \in \mathscr{E}, \forall t \in \mathscr{T} \\
& \forall i \in \mathscr{I}, \forall t \in \mathscr{T} \\
& P_{g}^{\min } x_{g}^{t} \leq \tilde{p}_{g}^{t} \leq P_{g}^{\max } x_{g}^{t} \\
& \forall g \in \mathscr{G}, \forall t \in \mathscr{T} \\
& \tilde{p}_{g}^{t}-\tilde{p}_{g}^{t-1} \leq R_{g}^{u} x_{g}^{t-1}+\tilde{R}_{g}^{u}\left(x_{g}^{t}-x_{g}^{t-1}\right)+P_{g}^{\max }\left(1-x_{g}^{t}\right) \quad \forall g \in \mathscr{G}, \forall t \in \mathscr{T} \\
& \tilde{p}_{g}^{t-1}-\tilde{p}_{g}^{t} \leq R_{g}^{d} x_{g}^{t}+\tilde{R}_{g}^{d}\left(x_{g}^{t-1}-x_{g}^{t}\right)+P_{g}^{\max }\left(1-x_{g}^{t-1}\right) \quad \forall g \in \mathscr{G}, \forall t \in \mathscr{T}
\end{aligned}
$$

The optimization objective (2a) is to minimize generation cost given a fixed unit commitment $\mathbf{x}$, where $C_{g}^{p}\left(\tilde{p}_{g}^{t}\right)$ is a linear approximation of generation cost for thermal units, as is commonly employed. We discuss this linearization further below. Constraints (2b)- $(2 \mathrm{~g})$ constitute an optimal power flow formulation, and include (in order): power balance at each bus (2b); power flow on a line, proportional to the difference in voltage phase angles at the terminal buses (2c); transmission line capacity limits (2d); lower and upper bounds for committed generation unit output levels (2e); and generation ramp-up/ramp-down constraints for pairs of consecutive time periods (2f) and (2g).

By linearizing the generation cost functions, (1)-(2) provides a mixed-integer linear programming (MILP) formulation of the unit commitment problem with transmission constraints, but without contingency constraints. A solution to the resulting BUC model provides an on/off schedule for all generation units, over all time periods in the scheduling horizon. In practice, committed generation units are adjusted on an hourly or sub-hourly basis, by ramping up or down specific units in order to 
satisfy realized demand. Further, additional fast-reaction (i.e., "peaker") units can be brought online if necessary. However, this process occurs on a different time scale than the BUC, e.g., one or two hours prior to real-time execution.

Remark 1 Our BUC model most closely represents the reliability unit commitment problem, which ISOs and vertically integrated utilities solve nightly. In contrast, the day-ahead unit commitment problem is executed earlier in the day, and is used to clear the market and set nodal electricity prices. While there are differences between the two problem variants, specifically in terms of the inputs (e.g., bids driving aggregate demand, in contrast to ISO-forecasted load), the basic BUC model can be easily recast into the day-ahead variant.

Remark 2 The number of time periods that unit $g$ has been online/offline prior to $t=1$ must satisfy $T_{g}^{u 0} \cdot T_{g}^{d 0}=0$. That is, if a generation unit $g$ is online prior to time period $1, T_{g}^{u 0}>0$ and $T_{g}^{d 0}=0$. Similarly, if unit $g$ is offline before time period 1 , $T_{g}^{u 0}=0$ and $T_{g}^{d 0}>0$.

Remark 3 The structure of the BUC solution space is known to be degenerate, due to the nature of the voltage phase angles $\tilde{\theta}_{i}^{t}$. In particular, alternative optimal solutions can be obtained by shifting all of the $\tilde{\theta}_{i}^{t}$ of a given optimal solution by a constant factor. To mitigate this degeneracy, and following common practice in the literature, we require in our numerical experiments that the value $\tilde{\theta}_{r}^{t}$ for a pre-defined "reference" bus $r$ be equal to 0 for all $t \in \mathscr{T}$.

Remark 4 Generation cost curves $C_{g}^{p}\left(p_{g}^{t}\right)$ are generally specified as quadratic functions of the form $C_{g}^{p}\left(p_{g}^{t}\right)=c_{g}^{p 2}\left(p_{g}^{t}\right)^{2}+c_{g}^{p 1} p_{g}^{t}+c_{g}^{p 0}$. However, because the $C_{g}^{p}\left(p_{g}^{t}\right)$ cost curves are non-decreasing convex functions of $p_{g}^{t}$, they can be easily approximated using a piecewise linear function (see [5]). Many researchers make a further simplification by assuming a linear cost function, which corresponds to the not uncommon case in which a generator offers into the market with a single marginal cost factor. We make this assumption below in our numerical experiments, specifically that $C_{g}^{p}\left(p_{g}^{t}\right)=c_{g}^{p} p_{g}^{t}$. The extension to the more general piecewise construct discussed above is straightforward, and does not impact the algorithms we introduce in Section 3. Practically, piecewise cost curves would inflate the solve times, but not significantly.

Remark 5 Variables and constraints to capture reserve requirements are common in the unit commitment literature, but are absent in our unit commitment models. As noted in [12][p. 1056], "The primary purpose of spinning and non-spinning reserves is to ensure there is enough capacity online to survive a contingency." Hedman et al. [12] make this argument in the context of $N-1$ reliabiliy; the argument for the exclusion of reserve models is even stronger for $N-k$ contingencies. Reserves, specifically spinning reserves, also serve as proxies for explicitly dealing with uncertainty in demand and variable generation (e.g., wind and solar plant) output. However, again following [12], we argue that enforcing $N-k$ reliability (even when $k=1$ ) is likely to ensure sufficient spinning reserves are online to deal with forecast errors in both demand and variable generation. We demonstrate that this is indeed the case in Section 2.3 by analyzing the CCUC for a 6-bus system. 


\subsection{N-k- $\varepsilon$ Contingency Constraints for Reliability Requirements}

According to the NERC TPL standard, in the event of a loss of a single component (i.e., an $N$-1 contingency), a power system must remain stable and satisfy all demand. In the case of two or more simultaneous losses (i.e., an $N-k$ contingency with $k \geq$ 2 ), the system must maintain stability. However, a pre-planned or controlled lossof-load is allowed. Therefore, prior to analyzing the contingency-constrained unit commitment problem, we must augment the BUC model to ensure that any resulting solutions can satisfy such requirements.

We consider the loss of elements in a power system $(\mathscr{I}, \mathscr{G}, \mathscr{E})$, considering both the set $\mathscr{G}$ of generation units and the set $\mathscr{E}$ of transmission lines. The additional parameters and the variables associated with our extension of the BUC formulation to include $N-k-\varepsilon$ are defined in Table 2.

Table 2 Variables and parameters $N-k-\varepsilon$ contingency analysis

\begin{tabular}{|c|c|}
\hline \multicolumn{2}{|l|}{ Parameters } \\
\hline$k$ & Maximum number of simultaneous element failures. \\
\hline $\mathscr{C}(j)$ & $\begin{array}{l}\text { Set of all contingencies with exactly } j \text { failed generation units } \\
\text { and/or transmission lines for } j \in\{1, \cdots, k\} \text {. Indexed by } c \text {. }\end{array}$ \\
\hline$|c|$ & Size of contingency $c$, i.e., the number of failed elements. \\
\hline $\mathscr{C}=\cup_{j=1}^{k} \mathscr{C}(j)$ & $\begin{array}{l}\text { Set of all contingencies with } k \text { or fewer failed elements (generation } \\
\text { units and/or transmission lines). }\end{array}$ \\
\hline$C$ & Index set of contingency set $\mathscr{C}$. \\
\hline$d_{g}^{c} \in\{0,1\}$ & $\begin{array}{l}\text { Parameter specifying whether generation unit } g \in \mathscr{G} \text { is involved in } \\
\text { contingency } c \in C \text {. }\end{array}$ \\
\hline$d_{e}^{c} \in\{0,1\}$ & $\begin{array}{l}\text { Parameter specifying whether transmission line } e \in \mathscr{E} \text { is involved in } \\
\text { contingency } c \in C \text {. }\end{array}$ \\
\hline $\mathbf{d}^{c} \in\{0,1\}^{G+T}$ & Vector representing the concatenation of $d_{g}^{c} \forall g \in \mathscr{G}$ and $d_{e}^{c} \forall e \in \mathscr{E}$. \\
\hline$\varepsilon_{j}$ & $\begin{array}{l}\text { Parameter indicating the maximum fraction of total system load that } \\
\text { can be shed in a size } j \text { contingency state, for } j=1, \cdots, k \text {. }\end{array}$ \\
\hline$\varepsilon$ & $\begin{array}{l}\text { Parameter vector indicating the maximum fraction of total load } \\
\text { that can be shed for each contingency size, i.e., } \varepsilon=\left(\varepsilon_{1}, \cdots, \varepsilon_{k}\right)\end{array}$ \\
\hline$\Delta_{g}^{j}$ & $\begin{array}{l}\text { Multiplicative factor applied to the ramping limits of generator } g \in \mathscr{G} \\
\text { during a size } j \in\{1, \cdots, k\} \text { contingency }\left(\Delta_{g}^{j} \geq 1\right) \text {. }\end{array}$ \\
\hline$\Delta_{e}^{j}$ & $\begin{array}{l}\text { Multiplicative factor applied to the power flow limits of transmission } \\
\text { line } e \in \mathscr{E} \text { during a size } j \in\{1, \cdots, k\} \text { contingency }\left(\Delta_{e}^{j} \geq 1\right) \text {. }\end{array}$ \\
\hline \multicolumn{2}{|l|}{ Variables } \\
\hline$p_{g}^{c t}, f_{e}^{c t}, \theta_{i}^{c t}$ & Corresponding values of $\tilde{p}_{g}^{t}, \tilde{f}_{e}^{t}, \tilde{\theta}_{i}^{t}$ during contingency $c \in \mathscr{C}$. \\
\hline$q_{i}^{c t}$ & Loss-of-load during contingency $c$ at bus $i$ at time $t$ \\
\hline
\end{tabular}

We express the $N-k$ contingency set $\mathscr{C}$ as follows:

$$
\mathscr{C}=\left\{\mathbf{d}^{c} \in\{0,1\}^{G+E}:\left(\sum_{g \in \mathscr{G}} d_{g}^{c}+\sum_{e \in \mathscr{E}} d_{e}^{c}\right) \leq k\right\} .
$$

Remark 6 The number of contingencies within the set $\mathscr{C}$ is given by:

$$
\left(\begin{array}{c}
G+E \\
1
\end{array}\right)+\cdots+\left(\begin{array}{c}
G+E \\
k
\end{array}\right) \leq(G+E+1)^{k}-1 .
$$


Practically, the number of contingencies grows so rapidly that explicit enumerationbased approaches are almost certain to fail even for modestly-sized systems.

We assume that a given contingency $c$ applies to all time periods $t \in \mathscr{T}$. For example, if a two-element contingency corresponds to the failure of elements $a$ and $b$, then these two elements are unavailable for all periods $t \in \mathscr{T}$. We are not modeling specific issues relating to when a contingency may occur, how long it may last, and what corrective measures may need to be taken to restore functionality. Such issues can significantly expand the size and difficulty of the associated unit commitment problem, and is beyond the scope of this work. Further, generation costs are not optimized in post-contingency operation; following precedence in the literature, only constraints related to power flow on the non-contingency system elements must be enforced. In other words, the primary goal during a contingency state is operational feasibility and not cost minimization. Additionally, multiple failure contingencies are extreme events with correspondingly low occurrence probabilities. Therefore, consideration of the production costs associated with these extreme events during operations planning is unnecessary, and may result in prohibitively expensive operations.

Given these assumptions, we now introduce the post-contingency corrective recourse constraints (i.e., the constraints that must be satisfied as the system state is altered in response to a contingency event, starting from a given steady state) $\mathscr{R}\left(\mathbf{x}, \tilde{\mathbf{p}}, \mathbf{d}^{c}\right)$ for a contingency prescribed by $\mathbf{d}^{c}$, under a unit commitment decision vector $\mathbf{x}$ and the no-contingency state generation schedule $\tilde{\mathbf{p}}$, as follows:

$$
\begin{array}{rlr}
\mathscr{R}\left(\mathbf{x}, \tilde{\mathbf{p}}, \mathbf{d}^{c}\right): & \sum_{g \in \mathscr{G}_{i}} p_{g}^{c t}+\sum_{e \in \mathscr{C}_{i}} f_{e}^{c t}-\sum_{e \in \mathscr{O}_{i .}} f_{e}^{c t}+q_{i}^{c t}=D_{i}^{t} & \forall i \in \mathscr{I}, \forall t \in \mathscr{T} \\
& B_{e}\left(\theta_{i_{e}}^{c t}-\theta_{j_{e}}^{c t}\right)\left(1-d_{e}^{c}\right)-f_{e}^{c t}=0 & \forall e \in \mathscr{E}, \forall t \in \mathscr{T} \\
& -F_{e} \Delta_{e}^{|c|}\left(1-d_{e}^{c}\right) \leq f_{e}^{c t} \leq F_{e} \Delta_{e}^{|c|}\left(1-d_{e}^{c}\right) & \forall e \in \mathscr{E}, \forall t \in \mathscr{T} \\
& p_{g}^{c t} \leq P_{g}^{\max } x_{g}^{t}\left(1-d_{g}^{c c}\right) & \forall g \in \mathscr{G}, \forall t \in \mathscr{T} \\
& p_{g}^{c t} \leq R_{g}^{u} \Delta_{g}^{|c|}+\tilde{p}_{g}^{t} & \forall g \in \mathscr{G}, \forall t \in \mathscr{T} \\
& -p_{g}^{c t} \leq\left(R_{g}^{d} \Delta_{g}^{|c|}-\tilde{p}_{g}^{t}\right)\left(1-d_{g}^{c}\right) & \forall g \in \mathscr{G}, \forall t \in \mathscr{T} \\
& q_{i}^{c t} \leq D_{i}^{t} & \forall i \in I, \forall t \in \mathscr{T} \\
& \sum_{i \in \mathscr{T}} q_{i}^{c t} \leq \varepsilon_{|c|} D^{t} & \forall t \in \mathscr{T} \\
& p_{g}^{c t} \geq 0 & \forall g \in \mathscr{G}, \forall t \in \mathscr{T} \\
& q_{i}^{c t} \geq 0 & \forall i \in \mathscr{I}, \forall t \in \mathscr{T} .
\end{array}
$$

Constraints (4a) enforce power balance at each bus, leveraging additional lossof-load variables $q^{c t}$. Constraints (4b) enforce DC power flow on those lines that are not part of contingency $\mathbf{d}^{c}$. Constraints (4c) enforce transmission line capacity limits. If a line is not part of a contingency, then the power flow limit is given by $F_{e} \Delta_{e}^{|c|}$; otherwise, the power flow is constrained to equal zero. Constraints (4d) enforce upper bounds on the power output of committed generation units not involved in the contingency $\mathbf{d}^{c}$; otherwise, the power output is constrained to be equal to zero. Constraints (4e) enforce generator ramp-up limits. If a generation unit is part of the 
Table 3 Generator data for the 6-bus test system

\begin{tabular}{c|c|cc|ccc}
\hline Unit & $\begin{array}{c}\text { Bus } \\
\text { No. }\end{array}$ & $\begin{array}{c}\text { Prod. } \\
(\$ / \mathrm{MW})\end{array}$ & $\begin{array}{c}\text { Start- } \\
\text { up }(\$)\end{array}$ & $\begin{array}{c}P^{\max } \\
(\mathrm{MW})\end{array}$ & $\begin{array}{c}P^{\mathrm{min}} \\
(\mathrm{MW})\end{array}$ & $\begin{array}{c}\text { Ramp } \\
(\mathrm{MW} / \mathrm{h})\end{array}$ \\
\hline G1 & 1 & 13.51 & 125 & 220 & 100 & 55 \\
G2 & 2 & 32.63 & 249 & 100 & 10 & 50 \\
G3 & 6 & 17.69 & 0 & 100 & 10 & 20 \\
G4 & 1 & 42 & 50 & 100 & 0 & 50 \\
G5 & 2 & 42 & 50 & 100 & 0 & 50 \\
G6 & 6 & 42 & 50 & 100 & 0 & 50 \\
\hline
\end{tabular}

contingency, then its corresponding power output level during the contingency is zero $\left(p_{g}^{c t}=0\right)$ and the constraint is non-binding. Otherwise, a generator can only ramp-up by $R_{g}^{u}$ from its pre-contingency level. Similarly, Constraints (4f) enforce generation ramp-down limits. If a generation unit is involved in a contingency, then $\left(1-d_{g}^{c}\right)=0$ and the ramp-down constraint is non-binding. Otherwise, a generator can only rampdown by $R_{g}^{d}$ from its pre-contingency level. Constraints (4g) ensure that loss-of-load at each bus does not exceed the demand at that bus. Finally, constraints (4h) ensure that at most $\varepsilon_{|c|}$ fraction of the aggregate demand can be shed in a size $|c|$ contingency.

Observe that in (4), lower limits on power output for generation units not in the contingency are relaxed to ensure sufficient operational flexibility. These lower limits can be easily incorporated for systems with sufficiently flexible generation units. In addition, we implicitly assume that the on/off state of generation units not involved in a contingency are fixed and cannot be changed via recourse variables during the contingency. For those generation units that are not involved in the contingency, the post-contingency power output levels $p_{g}^{c t}$ are not allowed to deviate from the baseline (pre-contingency) power output levels $\tilde{p}_{g}^{t}$ beyond the interval $\left[\tilde{p}_{g}^{t} R_{g}^{t}, \tilde{p}_{g}^{t}+R_{g}^{u}\right]$, given physical ramping limitations. In other words, $p_{g}^{c t} \in\left[\tilde{p}_{g}^{t} R_{g}^{t}, \tilde{p}_{g}^{t}+R_{g}^{u}\right]$.

Given the post-contingency corrective recourse constraints (4), the full CCUC formulation is given by combining formulations (1), (2), and (4).

\subsection{A 6-Bus Illustrative Example}

We now examine the impact of contingency constraints on optimal BUC solutions using the 6-bus test system introduced in [10]-[11]. Our goals are to concretely illustrate (a) the often significant changes in solution structure induced by the requirement to maintain $N-k-\varepsilon$ in unit commitment, relative to the baseline and $N-1$ cases, and (b) the redundant nature of contingency constraints, in that satisfaction of one contingency state yields solutions that can "cover" many other contingency states. The latter property is critical in the development of scalable algorithms for solving the CCUC, as we discuss in Section 3. The original test system consists of 6 buses, 7 transmission lines, and 3 generation units. We modified this instance for purposes of our analysis as follows. We augmented the system with three additional, fast-ramping generators G4, G5, and G6, located at buses 1, 2, and 6, respectively. This modification ensures there is sufficient generation capacity to satisfy the $N-k-\varepsilon$ criterion during contingency states. Data for the original generator set and the three additional generators is summarized in Table 3. Transmission line data is summarized in Table 4. 
Table 4 Transmission line data for the 6-bus test system

\begin{tabular}{c|c|c|c|c}
\hline $\begin{array}{c}\text { Line } \\
\text { No. }\end{array}$ & $\begin{array}{c}\text { From } \\
\text { Bus }\end{array}$ & $\begin{array}{c}\text { To } \\
\text { Bus }\end{array}$ & $B_{e}$ & $\begin{array}{c}F_{e} \\
(\mathrm{MW})\end{array}$ \\
\hline L1 & 1 & 2 & 5.88 & 200 \\
L2 & 1 & 4 & 3.88 & 100 \\
L3 & 2 & 4 & 5.08 & 100 \\
L4 & 5 & 6 & 7.14 & 100 \\
L5 & 3 & 6 & 55.56 & 100 \\
L6 & 2 & 3 & 27.03 & 100 \\
L7 & 4 & 5 & 27.03 & 100 \\
\hline
\end{tabular}

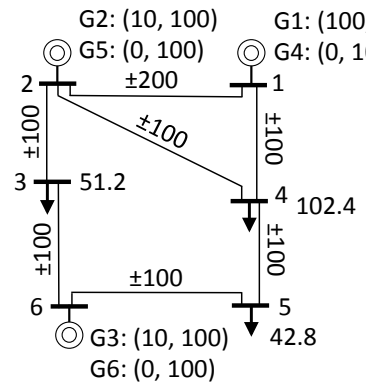

(a)

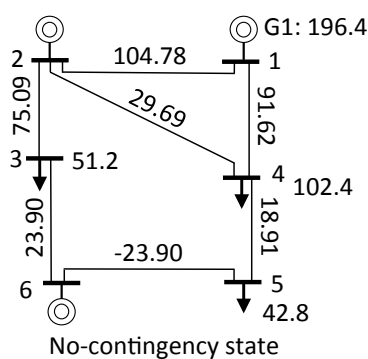

(b)

Fig. 1 (a) Single line diagram of the modified 6-bus test system. (b) An optimal BUC solution to the 6-bus test system, ignoring contingency constraints.

Consistent with [10]-[11], the unit shutdown cost is negligible and assumed to be zero in our analysis. For illustrative purposes only, we consider the BUC with a single time period, with loads of 51.2, 102.4, and 42.8 at buses 3,4 , and 5, respectively. Runtime results for the full 24-hour instance are presented in Section 4.

A single line diagram of the 6-bus test system is shown in Figure 1(a). Generator output bounds, transmission line capacity bounds, and loads are shown adjacent to their corresponding system elements. When contingencies are ignored, the optimal BUC solution commits a single unit (G1 at bus 1), generating 196.4 MW to meet the total demand. The no-contingency economic dispatch is shown graphically in Figure 1(b).

In accordance with NERC's TPL standard, loss-of-load is not permitted in singlecomponent-failure contingency states. In order for the 6-bus test system to be fully $N-1$ compliant, i.e., to operate the system in such a way that there exists a postcontingency corrective recourse action for all possible $N-1$ contingencies, 5 generation units must be committed, as shown in Figure 2(a). Of these, two units (G1 and G3) provide generation capacity during the no-contingency state, while three units (G4, G5, and G6) function as spinning reserves. Unlike the approach of explicitly setting aside spinning reserves (e.g., equivalent to the capacity of the largest on-line unit) via constraints, our proposed CCUC model implicitly and automatically selects 


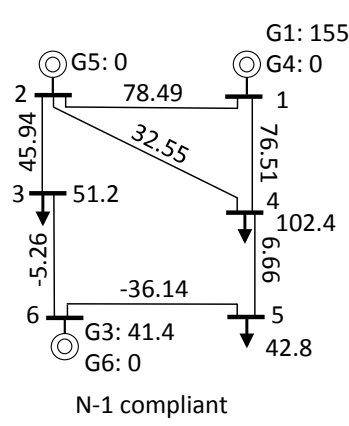

(a)

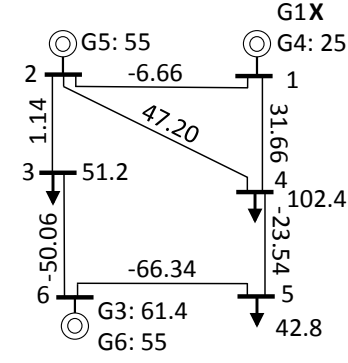

Post-contingency recourse (G1)

(b)

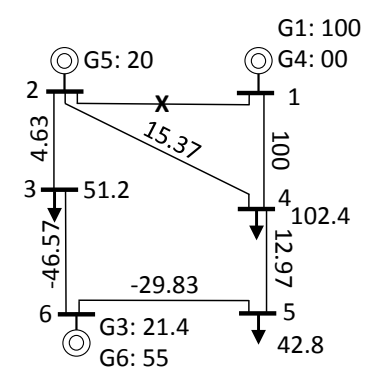

Post-contingency recourse (L1)

(c)

Fig. 2 (a) An optimal BUC solution to the 6-bus test system that is fully $N$-1 compliant. (b) A corrective recourse power flow after the failure of generation unit 1. (c) A corrective recourse power flow after the failure of the transmission line connecting buses 1 and 2 .

units to provide spinning reserves, by satisfying contingency constraints. Further, in contrast to the approach of explicitly allocating spinning reserves, our proposed CCUC model guarantees that there is adequate transmission capacity to dispatch the generator outputs during all contingency states.

The optimal $N$-1-compliant BUC solution shown in Figure 2(a) represents the system in steady state operations, i.e., under no observed contingency. Figures 2(b) and 2(c) illustrate feasible corrective recourse power flows for single-component contingency states corresponding to the failure of generation unit 1 and transmission line 1 (connecting buses 1 and 2), respectively. The total operating cost of the $N-1$ compliant solution is approximately $6.52 \%$ higher than an optimal no-contingency BUC solution.

The modified 6-bus system has 13 (7 transmission lines and 6 generators) possible single-component contingency states. We observe that it is sufficient to consider only the two contingency states shown in Figure 2(b) and Figure 2(c) in order to achieve full $N-1$ compliance. In other words, accounting for those two contingencies implicitly yields feasible corrective recourse actions for the other $N-1$ contingency states. As we discuss in Section 3, in most practical systems only a small number of contingency states are likely to impact the optimal unit commitment solution. Consequently, we design algorithms to screen for these critical contingencies implicitly, without the need to consider all possible combinations of system component failures - thus avoiding a combinatorial explosion in the number of possible contingencies.

If the maximum allowable contingency size is increased to $k=2$, the optimal BUC solution for the 6-bus test system commits four generation units, as shown in Figure 3 . In addition to including $k=2$ contingencies in our analysis, we require that loads must be fully served in the no-contingency state and that a post-contingency corrective resource action exists for all $k=1$ contingencies with no loss-of-load, per TPL standards. For all $k=2$ contingencies, the allowable loss-of-load threshold is set to $\varepsilon_{2}=0.27$, to ensure that there is sufficient slack to accommodate the losses of both 


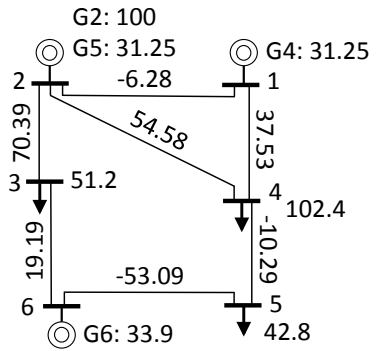

Fig. 3 An optimal BUC solution to the 6-bus test system that is fully $N-2-\varepsilon$-compliant, with $\varepsilon_{2}=0.27$ allowable loss-of-load factor.

transmission lines connected to bus 5 . If both transmission lines connected to bus 5 fail, then the load at that bus cannot be served; the factor 0.27 corresponds to the minimal loss-of-load under this contingency. For systems with greater redundancy and flexibility, such as those presented Section 4, the loss-of-load threshold can be set more conservatively (i.e., lower).

Of the four committed units, one unit (G1) is producing at maximum capacity and three units $(4,5$, and 6$)$ are producing at levels below their maximum rating. Taken together, these three units can provide up to $150 \mathrm{MW}$ of spinning reserves. Although fewer units are committed (4 compared to 5) relative to the $N$-1 solution, the two least expensive units (G1 and G2) are not committed while the three most expensive units (G4, G5, and G6) are committed in the $N-2-\varepsilon$ compliant solution.

We conclude with the obvious, yet critical, observation that contingency constraints must be considered in normal (no-contingency) unit commitment operations in order to ensure that a feasible post-contingency corrective recourse exists for all contingency states under consideration.

\subsection{Contingency-Constrained Unit Commitment Formulation}

Given the baseline unit commitment model (BUC) and associated contingency constraints defined respectively in Sections 2.1 and 2.2, we can now describe our full contingency-constrained unit commitment (CCUC) problem, as follows:

$$
\begin{aligned}
\text { CCUC : } \min _{\mathbf{x} \in \mathscr{X}} & \sum_{t \in \mathscr{T}} \sum_{g \in \mathscr{G}}\left(c_{g}^{u t}+c_{g}^{d t}\right)+\mathscr{Q}(\mathbf{x}) \\
\text { s.t. } & (\mathbf{f}, \mathbf{p}, \mathbf{q}, \boldsymbol{\theta}) \in \mathscr{R}\left(\mathbf{x}, \tilde{\mathbf{p}}, \mathbf{d}^{c}\right) \quad \forall c \in C
\end{aligned}
$$

The resulting unit commitment decision vector $\mathbf{x}$ represents a minimal-cost solution that satisfies (1) the non-contingency demands $D_{i}^{t}$ for each bus $i \in \mathscr{I}$ for each time period $t \in \mathscr{T}$, (2) the generation unit ramping constraints and startup/shutdown constraints, and (3) the network security and DC power flow constraints for each contingency, subject to loss-of-load allowances $\varepsilon_{j}$. We again note that generation costs incurred during a contingency are not considered in the objective function. Rather, only 
power system feasibility need be maintained, subject to the loss-of-load allowances $\varepsilon_{j}$, for all $j \in\{1, \cdots, k\}$.

\section{Solution Methods}

The explicit or extensive formulation (EF) (5) of the CCUC problem is a large-scale MILP, with an extremely large number of variables and constraints for even modest scale power systems and small contingency budgets $k$. For large-scale power systems and/or non-trivial contingency budgets $k$, the EF formulation will quickly become computationally intractable - assuming the resulting problem could be stored in memory, which is unlikely. For example, the number of constraints associated with feasibility checks in the CCUC (which drives the overall problem size) is approximately given as: $C \times T(3 I+2 E+4 G)=O\left(T \times(G+E)^{k} \times(I+G+E)\right)$.

The EF formulation of the CCUC problem has a structure that is amenable to a Benders decomposition (BD) approach, which partitions the constraints in the EF formulation into (1) a BUC problem prescribing the unit commitment decisions and the corresponding economic dispatch in the no-contingency state (this is commonly referred to as the master problem in BD), and (2) subproblems corresponding to feasibility checks, for each combination of contingency state $c \in \mathscr{C}$ and time period $t \in \mathscr{T}$. The BD algorithm iterates between solving the mixed-integer master problem (BUC), which prescribes the lowest cost unit commitment and economic dispatch, and the linear subproblems, until an optimal solution with a feasible post-contingency corrective recourse for all contingency states is obtained. In the following sub-section, we describe our Benders decomposition method, as it is applied to CCUC.

\subsection{A Benders Decomposition Approach}

We begin by observing that given a time period $t$, a unit commitment decision $\mathbf{x}^{t}$, and the no-contingency generation schedule $\tilde{\mathbf{p}}^{t}$, feasibility under contingency state $c$ with component failures defined by $\mathbf{d}^{c}$ is contingent on satisfying the corresponding standard DC power flow constraints. We refer to this problem as the contingency feasibility problem, which we denote $\mathbf{C F}\left(\mathbf{x}^{t}, \tilde{\mathbf{p}}^{t}, \mathbf{d}^{c}\right)$. For conciseness of notation, we eliminate the superscript " $c t$ " from the $f_{e}^{c t}, p_{g}^{c t}, q_{i}^{c t}$, and $\theta_{i}^{c t}$ decision variables. The 
$\mathbf{C F}\left(\mathbf{x}^{t}, \tilde{\mathbf{p}}^{t}, \mathbf{d}^{c}\right)$ constraint set is defined as follows:
( $\alpha) \sum_{g \in \mathscr{G}_{i}} p_{g}+\sum_{e \in \mathscr{E}_{i .}} f_{e}-\sum_{e \in \mathscr{E}_{i .}} f_{e}+q_{i}=D_{i}^{t}$
$\forall i \in \mathscr{I}$
(6a)
( $\beta) \quad B_{e}\left(\theta_{i_{e}}-\theta_{j_{e}}\right)\left(1-d_{e}^{c}\right)-f_{e}=0$
$\forall e \in \mathscr{E}$
$(\hat{\delta}) f_{e} \leq F_{e} \Delta_{e}^{|c|}\left(1-d_{e}^{c}\right)$
$\forall e \in \mathscr{E} \quad(6 \mathrm{c})$
$(\check{\delta}) \quad-f_{e} \leq F_{e} \Delta_{e}^{|c|}\left(1-d_{e}^{c}\right)$
$\forall e \in \mathscr{E} \quad(6 \mathrm{~d})$

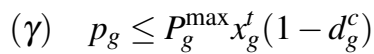
$\forall g \in \mathscr{G}$
(र) $\quad p_{g} \leq R_{g}^{u} \Delta_{g}^{|c|}+\tilde{p}_{g}^{t}$
$\forall g \in \mathscr{G}$
$(\check{\lambda}) \quad-p_{g} \leq\left(R_{g}^{d} \Delta_{g}^{|c|}-\tilde{p}_{g}^{t}\right)\left(1-d_{g}^{c}\right)$
$\forall g \in \mathscr{G} \quad(6 \mathrm{~g})$

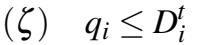
$\forall i \in \mathscr{I}$

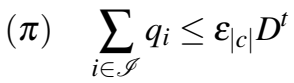

$$
p_{g}, q_{i} \geq 0
$$
$\forall g \in \mathscr{G}, i \in \mathscr{I}$.

Using the dual variables associated with each constraint set in (6a)-(6i), we observe (following from strong duality in linear programming) that $\left(\mathbf{x}^{t}, \tilde{\mathbf{p}}^{t}, \mathbf{d}^{c}\right)$ is feasible if and only if the following dual problem $\mathbf{D C F}\left(\mathbf{x}^{t}, \tilde{\mathbf{p}}^{t}, \mathbf{d}^{c}\right)$ is bounded:

$$
\begin{array}{rlr}
\max _{\alpha, \beta, \hat{\delta}, \check{\delta}, \gamma, \hat{\lambda}, \check{\lambda}, \zeta, \pi} & \sum_{i \in \mathscr{I}} D_{i}^{t}\left(\alpha_{i}+\zeta_{i}\right)+\sum_{e \in \mathscr{E}} F_{e} \Delta_{e}^{|c|}\left(1-d_{e}^{c}\right)\left(\check{\delta}_{e}+\hat{\delta}_{e}\right)+\sum_{g \in \mathscr{G}} P_{g}^{\max } x_{g}^{t}\left(1-d_{g}^{c}\right) \gamma_{g} \\
& +\sum_{g \in \mathscr{G}}\left(\left(R_{g}^{u} \Delta_{g}^{|c|}+\tilde{p}_{g}^{t}\right) \hat{\lambda}_{g}+\left(R_{g}^{d} \Delta_{g}^{|c|}-\tilde{p}_{g}^{t}\right)\left(1-d_{g}^{c}\right) \check{\lambda}_{g}+\varepsilon_{|c|} D^{t} \pi\right) \\
\text { s.t. } & \alpha_{i_{e}}-\alpha_{j_{e}}-\beta_{e}-\check{\delta}_{e}+\hat{\delta}_{e}=0 & \forall e \in \mathscr{E} \quad(7 \mathrm{~b}) \\
& \alpha_{i_{g}}+\gamma_{g}+\hat{\lambda}_{g}-\check{\lambda}_{g} \leq 0 & \forall g \in \mathscr{G} \quad(7 \mathrm{c}) \\
& \alpha_{i}+\zeta_{i} \leq 0 & \forall i \in \mathscr{I} \quad(7 \mathrm{~d}) \\
& \sum_{e \in \mathscr{E}_{i}} B_{e}\left(1-d_{e}^{c}\right) \beta_{e}-\sum_{e \in \mathscr{E}_{i}} B_{e}\left(1-d_{e}^{c}\right) \beta_{e}=0 & \forall i \in \mathscr{I} \quad(7 \mathrm{e}) \\
& \hat{\delta}, \check{\delta}, \gamma, \hat{\lambda}, \check{\lambda}, \zeta, \pi \leq 0 &
\end{array}
$$

Note that the feasible domain for $\mathbf{D C F}\left(\mathbf{x}^{t}, \tilde{\mathbf{p}}^{t}, \mathbf{d}^{c}\right)$ is a polyhedral cone and any solution in the domain is a ray. By Minkowski's theorem, every such ray can be expressed as a non-negative linear combination of the extreme rays of the domain. Therefore, the dual problem $\mathbf{D C F}\left(\mathbf{x}^{t}, \tilde{\mathbf{p}}^{t}, \mathbf{d}^{c}\right)$ is bounded if and only if its optimal objective value is less than or equal to zero. This condition holds if and only if:

$$
\begin{aligned}
& \sum_{i \in \mathscr{I}} D_{i}^{t}\left(\alpha_{i}+\zeta_{i}\right)+\sum_{e \in \mathscr{E}} F_{e} \Delta_{e}^{|c|}\left(1-d_{e}^{c}\right)\left(\check{\delta}_{e}+\hat{\delta}_{e}\right)+\sum_{g \in \mathscr{G}} P_{g}^{\max } x_{g}^{t}\left(1-d_{g}^{c}\right) \gamma_{g} \\
& +\sum_{g \in \mathscr{G}}\left(\left(R_{g}^{u} \Delta_{g}^{|c|}+\tilde{p}_{g}^{t}\right) \hat{\lambda}_{g}+\left(R_{g}^{d} \Delta_{g}^{|c|}-\tilde{p}_{g}^{t}\right)\left(1-d_{g}^{c}\right) \check{\lambda}_{g}+\varepsilon_{|c|} D^{t} \pi\right) \leq 0 .
\end{aligned}
$$


We call these constraints Benders feasibility cuts or $f$-cuts. Below, we summarize our Benders decomposition algorithm as applied to the CCUC, where $\mathrm{BUC}_{\ell}$ denotes the BUC formulation - in addition to any added $f$-cuts - at iteration $\ell$ of the algorithm:

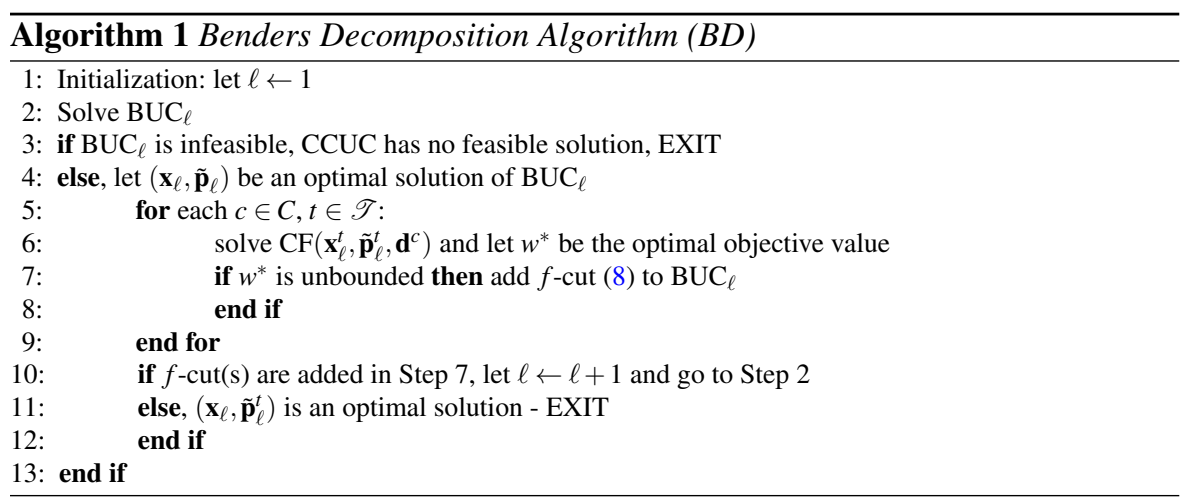

\subsection{A Cutting Plane Method Based on the Power System Inhibition Problem}

Even with a BD approach, solution of the CCUC is likely to be intractable for realistically sized power systems due to the number of feasibility checks that must be performed, one for each contingency $c \in C$ and time period $t \in \mathscr{T}$.

In this sub-section, we describe a cutting plane algorithm that uses a bi-level separation problem to implicitly identify a contingency state that would result in the worst-case loss-of-load for each contingency size $j, j \in\{1, \cdots, k\}$. If the worst-case generation shedding is non-zero and/or loss-of-load is above the given contingency load-shedding budget $\varepsilon_{j}$, then the current solution is infeasible. When such an infeasibility is detected, we generate a cutting plane (constraint) corresponding to $f$-cut (8), which is then added to the BUC master problem to protect against this particular contingency state.

\subsubsection{The Bi-Level Power System Inhibition Problem}

Given a time period $t \in \mathscr{T}$, a contingency budget $j \in\{1, \cdots, k\}$, a unit commitment $\mathbf{x}^{t}$, and the no-contingency generation levels $\tilde{\mathbf{p}}^{t}$, we solve a bi-level power system inhibition problem (PSIP), to determine the worst-case generation/load shedding under a contingency with exactly $j$ failed elements. In the context of the PSIP, the contingency vector $\mathbf{d}$ is no longer a parameter but a vector of upper-level decision variables. In the PSIP, the upper-level decisions (i.e., d) correspond to binary contingency selection decisions and the lower level decisions (i.e., f, p, q, r, $s, \theta$ ) correspond to the recourse generation schedule and DC power flow under the state prescribed by the 
the unit commitment decisions $\mathbf{x}^{t}$, the no-contingency state economic dispatch $\left(\tilde{\mathbf{p}}^{t}\right)$, and the upper-level contingency selection variables $(\mathbf{d})$.

Before we introduce the PSIP, we augment the previously introduced DC power flow constraints as follows. We introduce two sets of non-negative, continuous variables corresponding to generation shedding $r_{g}$ for all $g \in \mathscr{G}$ and loss-of-load at each bus $q_{i}$ for all $i \in \mathscr{I}$, and a single auxiliary variable $s$ corresponding to the total system loss-of-load above the allowable threshold $\varepsilon_{j} D^{t}$. In conjunction with associated constraints introduced below, these variables ensure that the PSIP has a feasible recourse power flow for any unit commitment $\mathbf{x}^{t}$, no-contingency state economic dispatch $\tilde{\mathbf{p}}^{t}$, and upper-level contingency selection decisions $\mathbf{d}$. We now formally state the PSIP, as follows (the "B" prefix below denotes "bi-level"):

$$
\begin{aligned}
& \operatorname{B-PSIP}\left(\mathbf{x}^{t}, \tilde{\mathbf{p}}^{t}, j\right) \text { : } \\
& \max _{\mathbf{d}} \min _{\mathbf{f}, \mathbf{p}, \mathbf{q}, \mathbf{r}, s, \theta} \sum_{g \in \mathscr{G}} r_{g}+s \\
& \text { s.t. } \quad \sum_{e \in \mathscr{E}} d_{e}+\sum_{g \in \mathscr{G}} d_{g}=j \\
& \sum_{g \in \mathscr{G}_{i}}\left(p_{g}-r_{g}\right)+\sum_{e \in \mathscr{E}_{. i}} f_{e}-\sum_{e \in \mathscr{E}_{i .}} f_{e}+q_{i}=D_{i}^{t} \\
& B_{e}\left(\theta_{i_{e}}-\theta_{j_{e}}\right)\left(1-d_{e}\right)-f_{e}=0 \quad \forall e \in \mathscr{E} \\
& -f_{e} \leq F_{e} \Delta_{e}^{j}\left(1-d_{e}\right) \\
& f_{e} \leq F_{e} \Delta_{e}^{j}\left(1-d_{e}\right) \\
& p_{g} \leq P_{g}^{\max } x_{g}^{t}\left(1-d_{g}\right) \\
& p_{g} \leq R_{g}^{u} \Delta_{g}^{j}+\tilde{p}_{g}^{t} \\
& -p_{g} \leq R_{g}^{d} \Delta_{g}^{j}-\tilde{p}_{g}^{t}\left(1-d_{g}\right) \\
& q_{i} \leq D_{i}^{t} \\
& r_{g}-p_{g} \leq 0 \\
& \forall i \in \mathscr{I}(9 \mathrm{c}) \\
& \forall e \in \mathscr{E}(9 \mathrm{e}) \\
& \forall e \in \mathscr{E} \text { (9f) } \\
& \forall g \in \mathscr{G} \\
& \forall g \in \mathscr{G} \\
& \forall i \in \mathscr{I} \\
& \sum_{i \in \mathscr{I}} q_{i}-s \leq \varepsilon_{j} D^{t} \\
& p_{g} \geq 0, q_{i} \geq 0, r_{g} \geq 0, s \geq 0 \\
& \forall i \in \mathscr{I}, g \in \mathscr{G} \\
& d_{e} \in\{0,1\}, d_{g} \in\{0,1\} \\
& \forall e \in \mathscr{E}, \forall g \in \mathscr{G}
\end{aligned}
$$

The bi-level objective (9a) seeks to maximize the minimum sum of (a) generation shedding and (b) loss-of-load quantity above the allowable threshold. Because both $r_{g}$ for all $g \in \mathscr{G}$ and $s$ are non-negative variables, the objective value is bounded from below by zero. If the objective value is equal to zero, the solution $\left(\mathbf{x}^{t}, \tilde{\mathbf{p}}^{t}\right)$ has a feasible corrective recourse for all contingencies of size $j$. Otherwise, the solution 
cannot survive the contingency prescribed by the upper-level contingency selection variables $\mathbf{d}$. Given a contingency state defined by $\mathbf{d}$, the objective of the power system operator (the inner minimization problem) is to find a corrective recourse power flow such that the sum of generation shedding and the loss-of-load quantity above the allowable threshold is minimized. Constraint (9b) enforces the budget on the number of power system elements in the selected contingency state. Constraints (9c) enforce power balance at each bus, with additional generation shedding variables $r_{g} \in \mathscr{G}$ for each generator located at a bus and a bus load-shedding variable $q_{i} \in \mathscr{I}$ to ensure system feasibility. Constraints (9d)-(9j) are as stated in formulation (4). Constraints (9k) restrict the amount of generation shedding to be at most the generation output for each generator $g \in \mathscr{G}$. Constraint (91) defines the amount of load shedding above the allowable threshold. If $\sum_{i \in \mathscr{I}} q_{i}>\varepsilon_{|c|} D^{t}$ then $s=\sum_{i \in \mathscr{I}} q_{i}-\varepsilon_{|c|} D^{t}$; otherwise, $s=0$.

Remark 7 Observe that Constraints (9d) are nonlinear, due to the presence of terms that are products of binary contingency-selection (upper level) variables and continuous voltage phase angles (lower level) variables. Thus, the B-SIP formulation is a bi-level, mixed-integer, non-linear program.

Remark 8 Observe that B-PSIP is feasible for any first-stage solution $\left(\mathbf{x}^{t}, \tilde{\mathbf{p}}^{t}\right)$ and any contingency prescribed by $\mathbf{d}$; the solution $\mathbf{f}=\mathbf{0}, \mathbf{p}^{t}=\mathbf{r}^{t}=\tilde{\mathbf{p}}^{t}, \mathbf{q}^{t}=D^{t}, s=\left(1-\varepsilon_{j}\right) D^{t}$ and $\boldsymbol{\theta}=\mathbf{0}$ is feasible under any contingency state.

Bi-level programs such as formulation (9) cannot in general be solved directly. Consequently, we next describe a reformulation strategy to derive a mixed-integer linear programming equivalent of the B-PSIP model. If the upper-level variables d are temporarily fixed, the inner minimization problem reduces to a linear program. By strong duality in linear programming, we can replace the inner minimization problem with its dual maximization problem. Subsequently releasing the temporarily fixed upper level variables $\mathbf{d}$ will yield a single-level, bi-linear program with bi-linear terms in the objective function. In the resulting reformulation, there are five nonlinear terms, specifically products of a binary contingency selection variable $\left(d_{e}, d_{g}\right)$ and a continuous dual variable $(\beta, \hat{\delta}, \check{\delta}, \gamma, \grave{\lambda})$. Each of these non-linear terms can be linearized using the following strategy.

Let $u$ and $v$ denote two continuous variables and let $b$ denote a binary variable. The bi-linear term $(1-b) u$ can then be linearized as follows. Letting $v=(1-b) u$, we introduce the following constraints to linearize the bi-linear term $(1-b) u$ :

$$
\begin{aligned}
u-U b & \leq v \leq u+U b \\
-U(1-b) & \leq v \leq U(1-b)
\end{aligned}
$$

Here, the parameter $U$ represents an upper bound for continuous variable $u$ that additionally satisfies $U \geq|u|$. Case analysis of these constraints for both values of $b$ demonstrates that they provide a linearization of the associated bi-linear term. If $b=1$, then Constraint (10b) implies that $v=0$. With $v=0$, Constraint (10a) implies that $-U \leq u \leq U$, which is never binding. If $b=0$, then Constraint (10a) implies $u=v$ and Constraint (10b) implies $-U \leq v \leq U$; the latter is never binding. 
Remark 9 If the bi-linear term is a product of a binary variable $b$ and a non-positive variable $u$, i.e. $u \leq 0$, the lower bound in Constraint (10b) is redundant, and can therefore be eliminated. Analogously, if $u$ is a non-negative variable, i.e. $u \geq 0$, the upper bound in Constraint (10b) is redundant, and can be eliminated.

We follow the above strategy to linearize all five bi-linear terms (involving $\beta, \hat{\delta}$, $\check{\delta}, \gamma$, and $\check{\lambda}$ ). First, we define the continuous variables $r^{1}, r^{2}, r^{3}, r^{4}$, and $r^{5}$, and let $r_{e}^{1}=\left(1-d_{e}\right) \beta_{e}, r_{e}^{2}=\left(1-d_{e}\right) \hat{\delta}_{e}, r_{e}^{3}=\left(1-d_{e}\right) \check{\delta}_{e}, r_{g}^{4}=\left(1-d_{g}\right) \gamma_{g}$, and $r_{g}^{5}=\left(1-d_{g}\right) \check{\lambda}_{g}$. For completeness, we present the full mixed-integer linear reformulation of the BPSIP formulation, as follows:

$$
\begin{aligned}
& \max _{\mathbf{d}, \alpha, \beta, \hat{\delta}, \breve{\delta}, \gamma, \hat{\lambda}, \check{\lambda}, \zeta, \pi} \sum_{i \in \mathscr{I}} D_{i}^{t}\left(\alpha_{i}+\zeta_{i}\right)+\sum_{e \in \mathscr{E}} F_{e} \Delta_{e}^{j}\left(r_{e}^{2}+r_{e}^{3}\right)+\sum_{g \in \mathscr{G}} P_{g}^{\max } x_{g}^{t} r_{g}^{4} \gamma_{g} \\
& +\sum_{g \in \mathscr{G}}\left(\left(R_{g}^{u} \Delta_{g}^{j}+\tilde{p}_{g}^{t}\right) \hat{\lambda}_{g}+\left(R_{g}^{d} \Delta_{g}^{j}-\tilde{p}_{g}^{t}\right) r_{g}^{5}+\varepsilon_{j} D^{t} \pi\right) \\
& \text { s.t. } \quad \sum_{e \in \mathscr{E}} d_{e}+\sum_{g \in \mathscr{G}} d_{g}=j \\
& \alpha_{i_{e}}-\alpha_{j_{e}}-\beta_{e}-\check{\delta}_{e}+\hat{\delta}_{e}=0 \\
& \forall e \in \mathscr{E} \quad(11 \mathrm{c}) \\
& \alpha_{i_{g}}+\gamma_{g}+\hat{\lambda}_{g}-\check{\lambda}_{g} \leq 0 \\
& \forall g \in \mathscr{G}(11 \mathrm{~d}) \\
& \alpha_{i}+\zeta_{i} \leq 0 \\
& \forall i \in \mathscr{I} \quad(11 \mathrm{e}) \\
& -\alpha_{i_{g}}+\eta_{g} \leq 1 \\
& \forall g \in \mathscr{G} \text { (11f) } \\
& -\pi \leq 1 \\
& \sum_{e \in \mathscr{E}_{i .}} B_{e} r_{e}^{1}-\sum_{e \in \mathscr{E}_{. i}} B_{e} r_{e}^{1}=0 \\
& r_{e}^{1} \geq \max \left\{\beta_{e}-U d_{e},-U\left(1-d_{e}\right)\right\} \\
& \forall i \in \mathscr{I}(11 \mathrm{~h}) \\
& r_{e}^{1} \leq \min \left\{\beta_{e}+U d_{e}, U\left(1-d_{e}\right)\right\} \\
& \forall e \in \mathscr{E} \quad \text { (11i) } \\
& r_{e}^{2} \geq \max \left\{\hat{\delta}_{e}-U d_{e},-U\left(1-d_{e}\right)\right\} \\
& \forall e \in \mathscr{E} \quad(11 \mathrm{j}) \\
& r_{e}^{2} \leq \hat{\delta}_{e}+U d_{e} \\
& \forall e \in \mathscr{E} \quad(11 \mathrm{k}) \\
& r_{e}^{3} \geq \max \left\{\check{\delta}_{e}-U d_{e},-U\left(1-d_{e}\right)\right\} \\
& \forall e \in \mathscr{E} \quad(111) \\
& r_{e}^{3} \leq \check{\delta}_{e}+U d_{e} \\
& \forall e \in \mathscr{E}(11 \mathrm{~m}) \\
& r_{g}^{4} \geq \max \left\{\gamma_{g}-U d_{g},-U\left(1-d_{g}\right)\right\} \\
& \forall e \in \mathscr{E} \quad(11 \mathrm{n}) \\
& r_{g}^{4} \leq \gamma_{g}+U d_{g} \\
& \forall g \in \mathscr{G} \text { (110) } \\
& r_{g}^{5} \geq \max \left\{\check{\lambda}-U d_{g},-U\left(1-d_{g}\right)\right\} \\
& \forall g \in \mathscr{G}(11 \mathrm{p}) \\
& r_{g}^{5} \leq \check{\lambda}+U d_{g} \\
& \forall g \in \mathscr{G} \text { (11q) } \\
& \hat{\delta}, \check{\delta}, \gamma, \hat{\lambda}, \check{\lambda}, \zeta, \pi \leq 0
\end{aligned}
$$

Next, we outline an algorithm for optimally solving the CCUC problem that combines a Benders decomposition approach with the aid of an oracle given by formulation (11), which acts as a separation problem. A given solution $\left(\mathbf{x}^{t}, \tilde{\mathbf{p}}^{t}\right)$ to the BUC is feasible with respect to $j$ if the oracle cannot find a contingency of size $j$ that results in a loss-of-load above the allowable threshold, i.e., if the optimal objective 
value is zero. For each contingency budget $j \in\{1, \cdots, k\}$, we can check for the worstcase $j$-element contingency by solving formulation (11) using a failure budget of $j$. Whenever the oracle determines that the current solution is not $N-k-\varepsilon$ compliant, it returns a contingency state prescribed by $\mathbf{d}$. This state results in generation shedding and/or loss-of-load above the allowable threshold $\varepsilon_{j} D^{t}$ for $j$-element failures.

\subsubsection{Contingency Screening Algorithms}

We now present our cutting plane algorithm, referred to as the Contingency Screening Algorithm 1 (CSA1), to solve the CCUC problem implicitly by screening for worstcase contingencies.

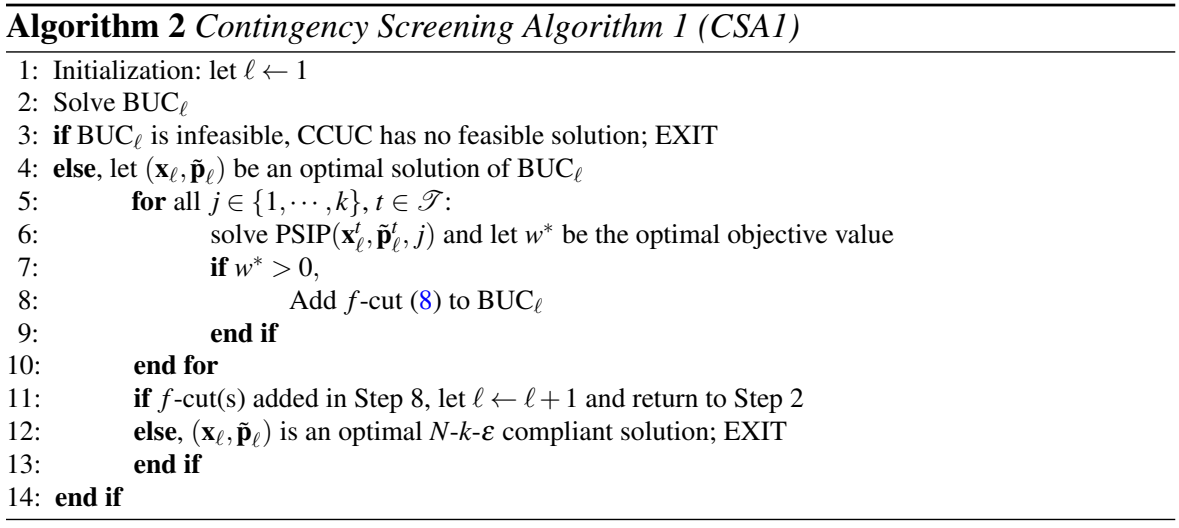

Remark 10 By employing algorithm CSA1, we have essentially recast the CCUC problem as a trilevel optimization problem. For related developments in tri-level power grid optimization, we defer to [32].

\subsubsection{Contingency Sharing Using a Dynamic Contingency List}

In preliminary testing using CSA1, we found that run time is significantly impacted by the need to solve a large number of PSIP instances at each master iteration of the algorithm. Specifically, we solve one instance of the PSIP for each maximum contingency budget and time period pair $(j, t)$, for each master iteration of the algorithm. The solution time of PSIP, as expected, is heavily impacted by the size of the power system $(\mathscr{I}, \mathscr{G}, \mathscr{E})$. Figure 4 shows the solution time (on a logarithmic scale) of the PSIP for various power system sizes and maximum contingency budgets $k$.

Based on our experience solving the CCUC problem using CSA1, we now make three observations regarding its behavior. First, the majority of the the total run time was spent solving PSIP instances. Second, a contingency $c$ that fails the system in one time period $t$ often fails the system in other time periods, suggesting that sharing of contingencies across time periods may be an effective mechanism to reduce 


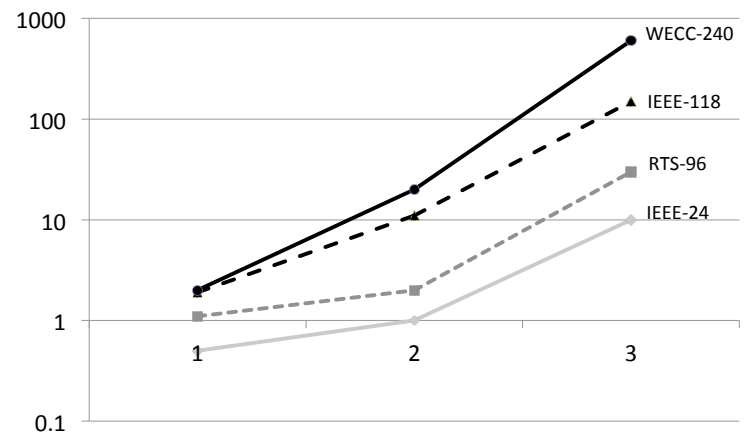

Fig. 4 Average run times (in seconds) of PSIP solves for varies power systems and contingency budgets $(k=1,2,3)$.

run times. Third, in the final solution only a small number of contingencies are actually identified. In other words, it is often prudent to consider a small number of contingencies explicitly when solving the CCUC problem. Based on these observations, we found that it is most efficient to develop a version of our CSA algorithm that minimizes the number times we solve PSIP instances and allows for sharing of contingencies across time periods. We achieve this by using a dynamic contingency list.

We begin with an empty contingency list $\mathcal{L}$. At each master iteration, we first screen all contingencies in the list for each time period $t \in \mathscr{T}$. For each time period $t$, we generate feasibility cuts (8) for each violated contingency in the list. If none of the contingencies in the list yields violations in any time period $t$, we proceed to solving PSIP instances to identify a new violated contingency. This simple procedure ensures that each violated contingency identified by solving PSIP is never redundant, i.e., the new contingency is not in our existing contingency list. When a new contingency is identified, we add it to the contingency list and check for its violation in all other time periods by solving a DC power flow problem.

Our computational results indicate that this procedure results in fewer total PSIP instances solved on average, which results in faster run times. The key idea behind this procedure is to avoid PSIP solves associated with re-identifying violated contingencies. We refer to this algorithm as the Contingency Screening Algorithm 2 (CSA2).

\section{Computational Experiments}

We implemented our proposed models and algorithms in C++ using IBM's Concert Technology Library 2.9 and the CPLEX 12.1 MILP solver. All experiments were performed on a workstation with two quad-core, hyper-threaded $2.93 \mathrm{GHz}$ Intel Xeon processors with $96 \mathrm{~GB}$ of memory. This yields a total of 16 threads allocated to each (mixed-integer) invocation of CPLEX. The default behavior of CPLEX 12.1 is to allocate a number of threads equal to the number of machine cores. In the case of hyper-threaded architectures, each core is presented as a virtual dual core - although 


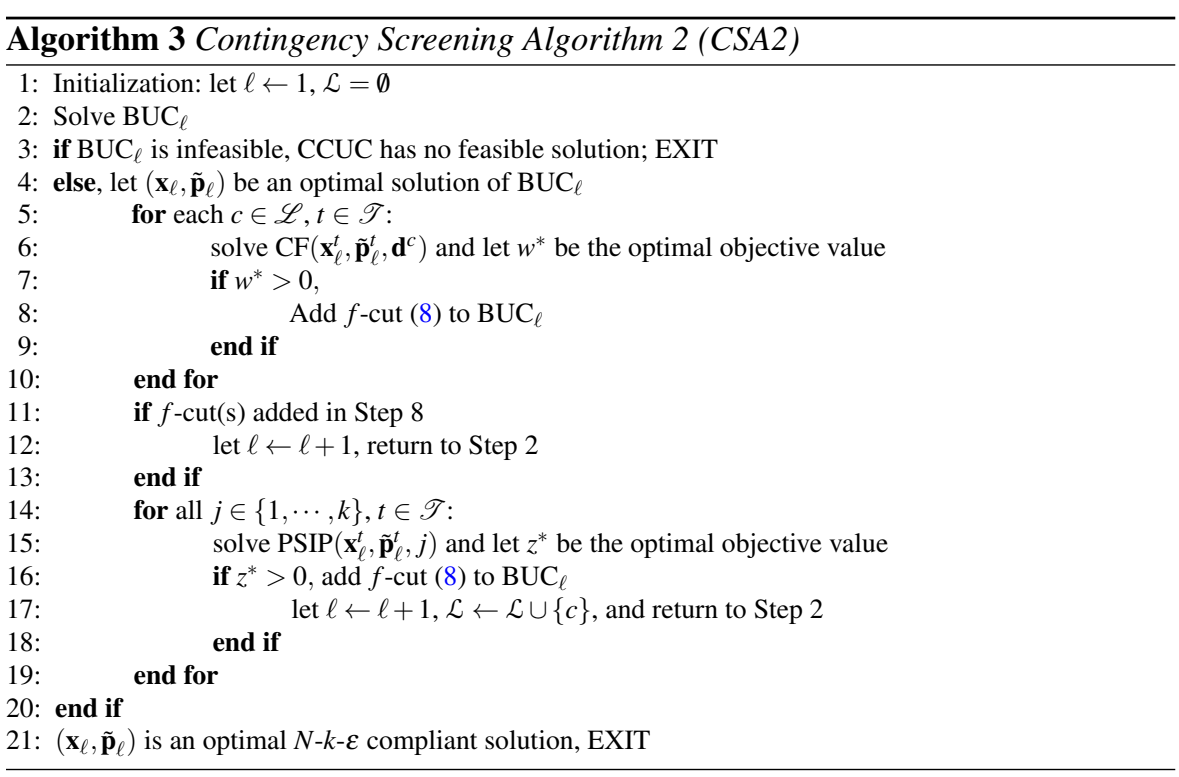

it is important to note that the performance is not equivalent to a true dual core. This workstation is shared by other users, such that our run time results should be interpreted as conservative. With the exception of the optimality gap, which we set to $0.1 \%$, we used the default CPLEX settings for all other parameters. All runs of all of our algorithms were allocated a maximum of 10,800 seconds ( 3 hours) of wall clock time.

We executed our models and algorithms on five test systems of varying size: the 6-bus, IEEE 24-bus, RTS-96, and IEEE 118-bus test systems [14], and a simplified model of the US Western interconnection (WECC-240) [23]. The 6-bus system - as described in Section 2.3 - is further augmented with three fast ramping generation units located at bus 1,2 , and 6 , respectively, to ensure there is sufficient generation capacity to respond to larger-size contingencies. Generator data for these three units are identical to G4-G6 in Table 3. To ensure there is sufficient operational flexibility in the WECC-240 system, we constrained transmission lines and one generation unit to be immune to failures. These nine elements include those serial lines, pairs of transmission lines, and generation unit and transmission line pairs whose failure would result in islanding of subsystems (buses). Additionally, we assume that nondispatchable generation injections into the system can be shed during contingency states. For each test system, we consider a 24 hour planning horizon and the four contingency budgets $k=0,1,2$, and 3, yielding a total of 20 instances.

We first consider the run times for our three different algorithms for solving the CCUC problem: the extensive form MILP, Benders decomposition, and the Contingency Screening Algorithm 2 (CSA2). The results are presented in Table 5. All times are reported in wall clock (elapsed) seconds. The column labeled " $C$ " reports the number of distinct contingencies for a given budget $k$, while the column labeled " $\varepsilon_{k}$ " reports the fraction of total load (demand) that can be shed. Entries in Table 5 report- 
Table 5 Run times for different solution approaches to the CCUC problem

\begin{tabular}{lccc|ccc}
\hline & & & & \multicolumn{3}{c}{ Solution time } \\
Test System & $C$ & $k$ & $\varepsilon_{k}$ & $\mathrm{EF}$ & $\mathrm{BD}$ & $\mathrm{CSA} 2$ \\
\hline 6-bus & 0 & 0 & 0 & 0 & 0 & 0 \\
& 16 & 1 & 0 & 3 & 2 & 1 \\
& 136 & 2 & 0.29 & 7 & 16 & 2 \\
& 696 & 3 & 0.77 & 134 & 189 & 4 \\
\hline IEEE 24-bus & 0 & 0 & 0 & 0 & 1 & 0 \\
& 70 & 1 & 0 & 108 & 58 & 11 \\
& 2,485 & 2 & 0.08 & $\mathrm{x}(\mathrm{LPR})$ & 3,861 & 101 \\
& 57,225 & 3 & 0.21 & $\mathrm{x}(\mathrm{OM})$ & $\mathrm{x}(0.03)$ & 397 \\
\hline RTS-96 & 0 & 0 & 0 & 1 & 2 & 2 \\
& 216 & 1 & 0 & $\mathrm{x}(\mathrm{LPR})$ & 303 & 41 \\
& 23,436 & 2 & 0.05 & $\mathrm{x}(\mathrm{OM})$ & 8,139 & 4,04 \\
& $1,679,796$ & 3 & 0.09 & $\mathrm{x}(\mathrm{OM})$ & $\mathrm{x}(0.050)$ & 4,989 \\
\hline IEEE 118-bus & 0 & 0 & 0 & 1 & 1 & 1 \\
& 240 & 1 & 0.01 & $\mathrm{x}(\mathrm{LPR})$ & 3,513 & 352 \\
& 28,920 & 2 & 0.12 & $\mathrm{x}(\mathrm{OM})$ & $\mathrm{x}(0.204)$ & 1,232 \\
& $2,304,200$ & 3 & 0.25 & $\mathrm{x}(\mathrm{OM})$ & $\mathrm{x}(0.249)$ & 8,586 \\
\hline WECC-240 & 0 & 0 & 0 & 1 & 2 & 2 \\
& 424 & 1 & 0 & $\mathrm{x}(\mathrm{LPR})$ & 262 & 108 \\
& 90,100 & 2 & 0.06 & $\mathrm{x}(\mathrm{OM})$ & $\mathrm{x}(0.004)$ & 2,484 \\
& $12,704,524$ & 3 & 0.15 & $\mathrm{x}(\mathrm{OM})$ & $\mathrm{x}(0.004)$ & $\mathrm{x}(0)$ \\
\hline
\end{tabular}

ing " $\mathrm{x}$ " indicate that the corresponding algorithm failed to locate an $N-k-\varepsilon$ compliant solution within the $0.1 \%$ optimality gap within the 3 hour time limit. For those instances that could not be solved within the allocated time, we provide exit status or feasibility gaps, indicating the maximum fraction of total demand shed above the allowable threshold $\varepsilon_{k}$ in the final solution. In all runs of the CSA2 algorithm, we initialize the contingency list $\mathcal{L}$ to the empty list.

As expected, the extensive form approach (EF) can only solve the smallest instances: for each contingency, a full set of DC power flow constraints must be explicitly embedded in the formulation. As the number of contingencies grows, this formulation quickly becomes intractable. The exit statuses "LPR" and "OM" represent "solving Linear Programming Relaxation at root node" and "Out of Memory," respectively. Note that our test instances only represent small to at most moderately sized systems relative to real power systems (which can contain on the order of thousands to tens of thousands of elements), indicating that even significant advances in solver technology are unlikely to mitigate this issue. Further, even given significant algorithmic advances, the memory requirements associated with the EF will likely cause the intractability to persist.

The BD approach attempts to address the exponential growth in the number of contingencies via delayed cut generation. However, although the BD approach does not explicitly incorporate DC power flow constraints for each contingency into the formulation, those power flow constraints must still be solved to identify violated feasibility cuts (which are then added to the master problem). In summary, the BD approach mitigates the memory issues associated with the EF approach, but the cost of identifying feasibility violations for a rapidly growing number of contingencies remains prohibitive. Overall, the $\mathrm{BD}$ approach can solve larger instances than the $\mathrm{EF}$ approach, but still fails given larger $k$ and larger test instances. 
Finally, we consider the performance of our third approach: CSA2. Here, we see that all of our test instances, with the sole exception of the WECC-240 system with $k=3$, can be solved within the 3 hour time limit. This result is enabled by the combination of using a dynamic contingency list (significantly reducing the number of PSIP solves) and the fact that we are able to implicitly evaluate all the contingencies in order to identify a violated contingency, and then quickly find a corresponding feasibility cut by solving a single linear program modeling DC power flow. These features of the CSA2 algorithm allow it to partially mitigate the impact of massive number of contingencies and the associated impact on run times and memory requirements. Lastly, we note that although CPA2 failed to solve the WECC-240 system with $k=3$ within the allocate time, the final solution at the three hour mark is in fact a $N-k-\varepsilon$ compliant solution. For large power systems and/or contingency budgets, significant computational time is required to "prove" feasibility. Eliminating the three hour time limit, we observed that the WECC-240 system with $k=3$ could be solved in approximately 18 hours, with the majority of this time taken to prove feasibility of the final solution.

We next examine the run times of our CSA2 algorithm in further detail, as reported in Table 6. For each instance, we report the total number of possible contingency states $C$ and the number of contingencies for which corresponding feasibility cuts were actually generated. The latter corresponds to the final size of the dynamic contingency list, which is reported in the column labeled "|L्L|." Clearly, $|\mathcal{L}|$ corresponds to a vanishingly small fraction of the possible number of contingencies, which is critical to the tractability of the approach. The remaining columns of Table 6 break down the total run time (in wall clock seconds) by the three main components of the algorithm - the RMP (Restricted Master Problem), which identifies unit commitments; the power system inhibition problem (PSIP), which identifies a contingency that has no feasible corrective recourse power flow given the current RMP UC decisions and no-contingency economic dispatch; and the contingency feasibility subproblems, which yield the feasibility cuts. The final column, labeled "cuts," reports the total number of feasibility cuts generated in solving the instance. It is clear from Table 6 that the computational bottleneck in the CSA2 algorithm is the solution of the PSIP, such that any improvements to that process will yield immediate reductions in CSA2 run times.

\section{Conclusions}

We have investigated the problem of scheduling generation units in power system operations, and determining a corresponding no-contingency state unit commitment and economic dispatch, such that the resulting solution satisfies the $N-k-\varepsilon$ reliability criterion. This reliability criterion is a generalization of the well-known $N-k$ criterion, and requires that at least $\left(1-\varepsilon_{j}\right)$ fraction of the total demand is met following the failure of $j$ system components, for $j \in\{1, \cdots, k\}$. We refer to this problem as the contingency-constrained unit commitment problem, or CCUC. We proposed two algorithms to solve the CCUC: one based on the Benders decomposition approach, and another based on contingency screening algorithms. The latter method avoids a 
Table 6 Run time breakout for the CSA2 algorithm

\begin{tabular}{lrrr|rrr|rrr}
\hline Test Systems & $C$ & $k$ & $\varepsilon_{k}$ & RMP & PSIP & DCF & itr & $|\mathcal{L}|$ & cuts \\
\hline 6-bus & 0 & 0 & 0 & 0 & 0 & 0 & 1 & 0 & 0 \\
& 16 & 1 & 0 & 0 & 1 & 0 & 2 & 1 & 21 \\
& 136 & 2 & 0.29 & 0 & 2 & 0 & 7 & 3 & 48 \\
& 696 & 3 & 0.77 & 0 & 4 & 0 & 11 & 4 & 89 \\
\hline IEEE 24-bus & 0 & 0 & 0 & 0 & 0 & 0 & 1 & 0 & 0 \\
& 70 & 1 & 0 & 6 & 46 & 1 & 185 & 1 & 185 \\
& 2,485 & 2 & 0.08 & 26 & 69 & 6 & 857 & 3 & 857 \\
& 57,225 & 3 & 0.21 & 64 & 324 & 9 & 928 & 4 & 928 \\
\hline RTS-96 & 0 & 0 & 0 & 2 & 0 & 0 & 1 & 0 & 0 \\
& 216 & 1 & 0 & 13 & 25 & 3 & 12 & 3 & 27 \\
& 23,436 & 2 & 0.05 & 17 & 385 & 2 & 15 & 4 & 33 \\
& $1,679,796$ & 3 & 0.09 & 19 & 4,965 & 5 & 17 & 5 & 38 \\
\hline WEEE 118-bus & 0 & 0 & 0 & 1 & 0 & 0 & 1 & 0 & 0 \\
& 240 & 1 & 0.01 & 243 & 72 & 37 & 85 & 5 & 1,305 \\
& 28,920 & 2 & 0.12 & 377 & 796 & 59 & 120 & 7 & 1,671 \\
& $2,304,200$ & 3 & 0.25 & 405 & 8,114 & 67 & 132 & 9 & 1,743 \\
\hline
\end{tabular}

combinatorial explosion in the number of contingencies by seeking vulnerabilities in the current solution, and generating valid inequalities to exclude such infeasible solutions in the master problem. We tested our proposed algorithms on test systems of varying sizes. Computational results show that our proposed Contingency Screening Algorithm (CSA2), which uses a bi-level separation program to implicitly consider all contingencies and a dynamic contingency list to avoid re-identification of contingencies, significantly outperforms the Benders decomposition approach. We were able to solve all test systems, with the exception of the largest WECC-240 instance, in under 3 hours. In contrast, both the Benders decomposition approach and direct solution of the CCUC extensive form fail to yield convergence on all but the smallest instances within 3 hours.

We believe that this paper will provide a significant basis for subsequent research in contingency-constrained unit commitment. For example, we are working to apply these methods to full-scale systems. While our results are promising in terms of scalability, full-scale problems pose more significant computational challenges, and consequently will require stronger formulations for the power system inhibition problem and possible adoption of high-performance computing resources. Further, our current CCUC model assumes all component failures occur simultaneously. In order to reflect practical operational situations, where failures may happen consecutively, new CCUC models that consider time lags between system component failures are needed. We plan to extend our CCUC models to include these cases. Finally, we have worked exclusively with a deterministic CCUC model to date. However, it is ultimately essential to incorporate uncertainty in our unit commitment models, e.g., to account for uncertain demand and renewable generation units. We believe our current cutting plane framework can be naturally extended to robust optimization and stochastic programming formulations via a nested decomposition approach. 
Acknowledgement. Sandia National Laboratories' Laboratory-Directed Research and Development Program and the U.S. Department of Energy's Office of Science (Advanced Scientific Computing Research program) funded portions of this work. Sandia National Laboratories is a multi-program laboratory managed and operated by Sandia Corporation, a wholly owned subsidiary of Lockheed Martin Corporation, for the U.S. Department of Energy's National Nuclear Security Administration under contract DE-AC04-94AL85000.

\section{References}

1. Arroyo, J.M. 2010. Bilevel programming applied to power system vulnerability analysis under multiple contingencies. IET Gener. Transm. Distrib. 4(2): 178-190.

2. Benders, J. 1962. Partitioning procedures for solving mixed-variables programming problems. $\mathrm{Nu}$ merische Mathematik 4: 238-252.

3. Bienstock, D., A. Verma. 2010. The N-k problem in power grids: new models, formulations, and numerical experiments. SIAM J. Optim. 20(5): 2352-2380.

4. Capitanescu, F., et al. 2011. State-of-the-art, challenges, and future trends in security constrained optimal power flow. Elec. Power Syst. Res. 81: 1731-1741.

5. Carrión, M., J.M. Arroyo. 2006. A compuationally efficient mixed-integer linear formulation for the thermal unit commitment problem. IEEE Trans. Power Syst. 21(3): 1371-1378.

6. Chen, R.L., A. Cohn, N. Fan, A. Pinar. 2012. $N-k-\varepsilon$ power system design. Proc. 12th Probabilistic Methods for Power Syst. Conf.. Istanbul, Turkey.

7. Chen, R.L., A. Cohn, N. Fan, A. Pinar. 2014. Contingency-risk informed power system design. IEEE Trans. Power Systems. DOI: 10.1109/TPWRS.2014.2301691.

8. Fan, N., H. Xu, F. Pan, P.M. Pardalos. 2011. Economic analysis of the N-k power grid contingency selection and evaluation by graph algorithms and interdiction methods. Energy Syst. 2(3-4): 313-324.

9. FERC Staff Report. Recent ISO Software Enhancements and Future Software and Modeling Plans. Federal Energy Regulatory Commission.

10. Fu, Y., M. Shahidehpour, Z. Li. 2005. Security-constrained unit commitment with AC constraints. IEEE Trans. Power Syst. 20(3): 1538-1550.

11. Fu, Y., M. Shahidehpour, Z. Li. 2006. AC contingency dispatch based on security-constrained unit commitment. IEEE Trans. Power Syst. 21(2): 897-908.

12. Hedman, K.W., M.C. Ferris, R.P. O'Neill, E.B. Fisher, S.S. Oren. 2010. Co-optimization of generation unit commitment and transmission switching with N-1 reliability. IEEE Trans. Power Syst. 24(2): $1052-1063$.

13. Hobbs, B. F., M. H. Rothkopf, R. P. O’Neill, H.-P. Chao, eds. 2001. The Next Generation of Electric Power Unit Commitment Models. Kluwer Academic Publishers, Boston.

14. IEEE Test Systems. http://www.ee.washington.edu/research/pstca.

15. Lesieutre B., S. Roy, V. Donde, and A. Pinar, Power system extreme event analysis using graph partitioning, Proc. 39th North American Power Symposium, Carbondale, IL, October 2006.

16. Lesieutre B., A. Pinar, and S. Roy, Power System Extreme Event Detection: The Vulnerability Frontier, in Proc. 41st Hawaii International Conference on System Sciences, pages 184, Waikoloa, Big Island, HI, 2008.

17. Lotfjou, A., M. Shahidehpour, Y. Fu, Z. Li. 2010. Security-constrained unit commitment with AC/DC transmission systems. IEEE Trans. Power Syst. 25(1): 531-542.

18. North American Electric Reliability Corporation, Transmission Planning Standards, Accessed on April 2014. Available at http://www.nerc.com/pa/Stand/Reliability\%20Standards/Forms/AllItems.aspx

19. O'Neill, R.P., K.W. Hedman, E.R. Krall, A. Papavasiliou, S.S. Oren. 2010. Economic analysis of the $\mathrm{N}-1$ reliable unit commitment and transmission swtiching problem using duality concepts. Energy Syst. 1)(2): 165-195

20. Personal Communication. Dr. Eugene Litvinov. March, 2012.

21. Padhy, N.P. 2004. Unit commitment - A bibliographical survey. IEEE Trans. Power Syst. 19(3): 11961205.

22. Pinar, A., J. Meza, V. Donde, B. Lesieutre. 2010. Optimization strategies for the vulnerability analysis of the electric power grid. SIAM J. Optim. 20(4): 1786-1810. 
23. Price, J.E. 2011. Reduced Network Modeling of WECC as a Market Design Prototype. Proceedings of the 2011 IEEE General Meeting of the Power and Energy Society. San Diego, California.

24. Salmeron, J., K. Wood, R. Baldick. 2004. Analysis of electric grid security under terrorist threat. IEEE Trans. Power Syst. 19(2): 905-912.

25. Salmeron, J., K. Wood, R. Baldick. 2009. Worst-case interdiction analysis of large-scale electric power grids. IEEE Trans. Power Syst. 24(1): 96-104.

26. Street, A., F. Oliveira, J.M. Arroyo. 2011. Contingency-constrained unit commitment with n-K security criterion: A robust optimization approach. IEEE Trans. Power Syst. 26(3): 1581-1590.

27. Street, A., F. Oliveira, J.M. Arroyo. 2011. Energy and reserve scheduling under an N-K security criterion via robust optimization. Proc. 17th Power Syst. Compt. Conf.. Stockholm, Sweden.

28. Wang, J., M. Shahidehpour, Z. Li. 2008. Security-constrained unit commitment with volatile wind power generation. IEEE Trans. Power Syst. 23(3): 1319-1327.

29. Wang, Q., J.-P. Watson, Y. Guan. 2013. Two-stage robust optimization for $N-k$ contingencyconstrained unit commitment. IEEE Trans. Power Syst. 28(3): 2366-2375.

30. Wood, A.J., Wollenberg, B.J., 1996. Power Generation, Operation and Control (2nd edition). John Wiley \& Sons, New York.

31. Wu, L., M. Shahidehpour. 2010. Accelerating the Benders decomposition for network-constrained unit commitment problems. Energy Syst. 1(3): 339-376.

32. Yuan, W., L. Zhao, B. Zeng. 2014. Optimal power grid protection through a defender-attackerdefender model. Reliability Engineering and System Safety 121: 83-89.

33. Zhao, L., B. Zeng. 2013. Vulnerability analysis of power grids with line switching. IEEE Transactions on Power Syst. 28(3): 2727-2736.

34. Zheng, Q.P., J. Wang, P.M. Pardalos, Y. Guan. 2013. A decomposition approach to the two-stage stochastic unit commitment problem. Annals of Operations Research 210(4): 387-410. 